\title{
HOMOMORPHISMS AND ENDOMORPHISMS IN VARIETIES OF PSEUDOCOMPLEMENTED DISTRIBUTIVE LATTICES (WITH APPLICATIONS TO HEYTING ALGEBRAS)
}

\author{
$\mathrm{BY}$
}

\author{
M. E. ADAMS, V. KOUBEK ${ }^{1}$ AND J. SICHLER ${ }^{1}$
}

\begin{abstract}
According to a result by K. B. Lee, the lattice of varieties of pseudocomplemented distributive lattices is the $\omega+1$ chain $B_{-1} \subset B_{0} \subset$ $B_{1} \subset \cdots \subset B_{n} \subset \cdots \subset B_{\omega}$ in which the first three varieties are formed by trivial, Boolean, and Stone algebras respectively. In the present paper it is shown that any Stone algebra is determined within $B_{1}$ by its endomorphism monoid, and that there are at most two nonisomorphic algebras in $B_{2}$ with isomorphic monoids of endomorphisms; the pairs of such algebras are fully characterized both structurally and in terms of their common endomorphism monoid. All varieties containing $B_{3}$ are shown to be almost universal. In particular, for any infinite cardinal $\kappa$ there are $2^{\kappa}$ nonisomorphic algebras of cardinality $\kappa$ in $B_{3}$ with isomorphic endomorphism monoids. The variety of Heyting algebras is also almost universal, and the maximal possible number of nonisomorphic Heyting algebras of any infinite cardinality with isomorphic endomorphism monoids is obtained.
\end{abstract}

1. Introduction. Endomorphisms of any algebra $A$ form a monoid $\operatorname{End}(A)$ under composition; the present paper is concerned with endomorphisms of distributive lattices.

The dual $L^{d}$ of a distributive lattice $(L ; \vee, \wedge)$ is the distributive lattice $(L ;+, \cdot)$ for which $x+y=x \wedge y$ and $x \cdot y=x \vee y$ for all $x, y \in L$. Clearly, $\operatorname{End}\left(L^{d}\right) \cong \operatorname{End}(L)$ for any distributive lattice although, in general, $L^{d}$ and $L$ need not be isomorphic. In B. M. Schein [46], however, it is shown that a distributive lattice is determined up to its dual by its endomorphism monoid. Similarly, only a distributive $(0,1)$ lattice and its dual have isomorphic $(0,1)$-endomorphism monoids (R. McKenzie and $\mathrm{C}$. Tsinakis [37]). On the other hand, distributive 0-lattices or 1-lattices are determined by monoids of their respective bound-preserving endomorphisms [45].

Boolean algebras, that is, distributive lattices with complementation as an added unary operation, are once again determined by their endomorphism monoids (K. D. Magill [34], B. M. Schein [46], C. J. Maxson [35]). Boolean algebras are particular examples of pseudocomplemented distributive lattices, the present topic of investigation.

A pseudocomplemented distributive lattice $(L ; \vee, \wedge, *, 0,1)$ is an algebra of type $(2,2,1,0,0)$, where $(L ; \vee, \wedge, 0,1)$ is a distributive $(0,1)$-lattice and $*$ is the unary operation of pseudocomplementation: the pseudocomplement $x^{*}$ of $x \in L$ satisfies $x \wedge y=0$ if and only if $y \leq x^{*}$ for every $y \in L$. It appears that pseudocomplemented distributive lattices were studied first by V. Glivenko in [15];

Received by the editors April 28, 1983.

1980 Mathematics Subject Classification. Primary 06D15, 06D20; Secondary 08A35, 18 B15.

${ }^{1}$ The support of NSERC is gratefully acknowledged. 
sometime later, P. Ribenboim [45] proved that pseudocomplemented distributive lattices form a variety. Subsequently, K. B. Lee [33] fully described the lattice of all varieties of pseudocomplemented distributive lattices; the lattice is an $\omega+1$ chain $B_{-1} \subset B_{0} \subset B_{1} \subset \cdots \subset B_{\omega}$. The variety $B_{-1}$ consists of all one-element algebras and $B_{0}$ is the variety of all Boolean algebras; for $n \geq 1, \mathrm{~K}$. B. Lee showed that the variety $B_{n}$ is defined by the identity

$$
\left(x_{1} \wedge \cdots \wedge x_{n}\right)^{*} \vee\left(x_{1}^{*} \wedge \cdots \wedge x_{n}\right)^{*} \vee \cdots \vee\left(x_{1} \wedge \cdots \wedge x_{n}^{*}\right)^{*}=1
$$

Three important papers quickly followed this discovery (namely G. Grätzer and H. Lakser $[\mathbf{1 8}, \mathbf{1 9}]$, and H. Lakser [32]), and more since then; see R. Balbes and $\mathrm{Ph}$. Dwinger [3] for numerous references on this subject. In fact, the variety $B_{1}$ of Stone algebras had already been intensively studied by G. Grätzer and E. T. Schmidt [21], J. C. Varlet [49] and others.

The present paper investigates the question of determining a pseudocomplemented distributive lattice from its endomorphism monoid.

In $\S 3$ it will be shown that the structure of $\operatorname{End}(L)$ determines whether or not the pseudocomplemented distributive lattice $L$ has a homomorphic image in $B_{1} \backslash B_{0}$; anticipating this result, let us call $\operatorname{End}(L)$ a Stone monoid if $L$ has a Stone homomorphic image that is not Boolean. Thus, for instance, $\operatorname{End}(L)$ is a Stone monoid for every $L \in B_{1} \backslash B_{0}$. The situation is described by the following

THEOREM 1.1. (i) If $L \in B_{2}$ and if $\operatorname{End}(L)$ is a Stone monoid, then $L$ is determined within $B_{2}$ by $\operatorname{End}(L)$.

(ii) For $L \in B_{2}$ such that $\operatorname{End}(L)$ is not a Stone monoid there exists at most one algebra $L^{+} \in B_{2}$ not isomorphic to $L$ and such that $\operatorname{End}\left(L^{+}\right) \cong \operatorname{End}(L)$.

(iii) The varieties $B_{3}, B_{4}, \ldots, B_{\omega}$ are almost universal.

Observe that Theorem 1.1(i) implies that Stone algebras are uniquely determined by their endomorphism monoids, thereby extending the earlier quoted result on Boolean algebras to the variety $B_{1}$. Nonisomorphic algebras $L, L^{+} \in B_{2}$ with isomorphic endomorphism monoids are fully characterized in $\S 3$ of the present paper. Almost universality (defined below) of $B_{3}, B_{4}, \ldots, B_{\omega}$ implies that, in particular, algebras from each of these varieties with isomorphic endomorphism monoids bear very little structural similarity to one another.

A homomorphism $f: L \rightarrow L^{\prime}$ of pseudocomplemented distributive lattices will be called constant if $f(L)=\{0,1\} \subseteq L^{\prime}$, that is, if its image is the set of constants of its codomain. Every nontrivial pseudocomplemented distributive lattice has a minimal prime ideal $I$, and the mapping defined by $f(I)=\{0\}, f(L \backslash I)=\{1\}$ is a homomorphism of $L$ onto the two-element chain $\{0,1\}$ (see [3]); in other words, there are constant homomorphisms between any pair of pseudocomplemented distributive lattices. A variety $V$ is almost universal if the category of all undirected graphs and all their compatible mappings is isomorphic to a subcategory of $V$ consisting of all nonconstant homomorphisms between algebras from some subclass $D$ of $V$. Thus nonconstant homomorphisms between algebras in $D$ must form a category; that is, the composite of nonconstant homomorphisms must never be constant. Almost universal varieties possess a very rich categorical structure (see A. Pultr and V. Trnková [43]). From Theorem 1.1(iii) it will follow, for instance, that for every infinite cardinal $\kappa$ there exists a system $\left(L_{i} \in B_{3}: i \in 2^{\kappa}\right)$ such that $\operatorname{Hom}\left(L_{i}, L_{j}\right)$ consists of constants for distinct $i, j \in 2^{\kappa}$, while $\left|L_{i}\right|=\kappa$, and $\operatorname{End}\left(L_{i}\right)$ 
is isomorphic to the monoid of $2^{\kappa}$ right zeros and the identity endomorphism for every $i<2^{\kappa}$.

If the category of all isomorphisms of undirected graphs is isomorphic to the category of all isomorphisms between algebras from a subclass $D$ of a variety $V$, we will say that $V$ is isomorphism universal. For every group $G$ there is a proper class $\left(A_{i} \in V: i \in I\right)$ of pairwise nonisomorphic algebras in any isomorphism universal variety $V$ such that $\operatorname{Aut}\left(A_{i}\right) \cong G$ for every $i \in I$ (see [26]). While the variety $B_{0}$ of Boolean algebras is not isomorphism universal (R. McKenzie and J. D. Monk [36]), Corollary 4.18 below shows that all other subvarieties of $B_{\omega}$ are isomorphism universal.

As it happens, the construction used to prove Theorem 1.1(iii) produces distributive $(0,1)$-lattices that are Heyting algebras as well. Recall that an algebra $(H ; \vee, \wedge, \rightarrow, 0,1)$ of type $(2,2,2,0,0)$ is a Heyting algebra if $(H ; \vee, \wedge, 0,1)$ is a distributive $(0,1)$-lattice with an added operation $\rightarrow$ of relative pseudocomplementation defined by $z \leq x \rightarrow y$ just when $x \wedge z \leq y$. The class of all Heyting algebras is a variety (see [3] or H. Rasiowa and R. Sikorski [44]). A homomorphism $f: H \rightarrow H^{\prime}$ of Heyting algebras is a mapping preserving all five operations; in particular, $f$ is a $(0,1)$-homomorphism of the underlying distributive lattices. Any nontrivial Heyting algebra $H$ has a minimal prime ideal and, as before, the characteristic function of the complementary prime filter is a constant homomorphism of $H$ onto the set $\{0,1\}$ of constants of any nontrivial Heyting algebra $H^{\prime}$. There are no nonconstant extraneous Heyting algebra homomorphisms, however; in fact, the following will be shown to hold.

\section{THEOREM 1.2. The variety of Heyting algebras is almost universal.}

One of the consequences of this result is that for any infinite cardinal $\kappa$ there is a family $\left(H_{i}: i \in 2^{\kappa}\right)$ of Heyting algebras such that $\left|H_{i}\right|=\kappa$ for all $i \in 2^{\kappa}$, $\operatorname{Hom}\left(H_{i}, H_{j}\right)$ consists of $2^{\kappa}$ constant maps if $i, j \in 2^{\kappa}$ are distinct, and, for all $i \in 2^{\kappa}, \operatorname{End}\left(H_{i}\right)$ is isomorphic to the monoid obtained by adding $2^{\kappa}$ right zeros to the trivial monoid. The latter claim shows that a Heyting algebra is not determined by its endomorphism monoid, thereby solving a problem posed, for instance, by R. McKenzie and C. Tsinakis in [37]. Observe that Theorem 1.2 also proves the isomorphism universality of the variety of Heyting algebras. In a subsequent paper it will be shown that the number of right zeros of $\operatorname{End}(H)$ can be reduced to one, the least number possible.

Priestley's duality [39] between distributive $(0,1)$-lattices and certain ordered topological spaces will be used throughout the paper; the results will also be interpreted algebraically.

With pleasure we acknowledge correspondence with $\mathrm{R}$. Beazer on the subject of pseudocomplemented distributive lattices as well as conversations with $\mathrm{C}$. Tsinakis which led to the investigations presented here.

2. Preliminaries. This section provides the minimal background and notation concerning pseudocomplemented distributive lattices and Priestley's duality applicable to these lattices. For further information on pseudocomplemented distributive lattices the reader is referred to $[\mathbf{3}]$ or G. Grätzer $[\mathbf{1 6}, \mathbf{1 7}]$. Fuller description of the topological duality can be found in H. A. Priestley $[39,40]$; see also the more recent survey articles by B. A. Davey and D. Duffus [12] or H. A. Priestley [42]. 
H. Lakser [32] showed that a pseudocomplemented distributive lattice is subdirectly irreducible if and only if as a lattice it is isomorphic to a Boolean lattice with a new unit added. The variety $B_{n}$ is then generated by the finite subdirectly irreducible lattice with $n$ atoms if $n>0$, while $B_{0}$ is the variety of Boolean algebras (see K. B. Lee $[\mathbf{3 3}]$ ).

Proposition 2.1 (K. B. LEE $[33]$ ). For any finite $n>0$, a pseudocomplemented distributive lattice $L$ lies in $B_{n}$ if and only if each prime ideal of $L$ contains at most $n$ minimal prime ideals.

Next is a brief summary of Priestley's duality as it applies to pseudocomplemented distributive lattices.

For a poset $P$, a subset $Q$ of $P$ is decreasing if $x \in P, x \leq q \in Q$ imply $x \in Q$. A mapping $f: P \rightarrow P^{\prime}$ is order preserving if $f(x) \leq f(y)$ in $P^{\prime}$ whenever $x \leq y$ in $P$. Endowed with a topology $\tau$, the pair $(P, \tau)$ is a totally order disconnected space if for all $x, y \in P$ with $x \nsupseteq y$ there exists a clopen decreasing $Q \subseteq P$ such that $x \in Q$ and $y \in P \backslash Q$.

TheOREM 2.2 (H. A. PRIESTLEy $[39,40]$ ). The category of all $(0,1)$ homomorphisms of distributive $(0,1)$-lattices is dually isomorphic to the category $T$ of all continuous order preserving mappings of compact totally order disconnected spaces.

In fact, if $(P, \tau) \in T$ and a lattice $L$ correspond in this duality, then $P$ is the poset of all prime ideals of $L$ ordered by inclusion, while elements of $L$ are represented by clopen decreasing subsets of $(P, \tau)$. Furthermore, if $\left(P^{\prime}, \tau^{\prime}\right)$ corresponds to $L^{\prime}$ and if $h: L \rightarrow L^{\prime}$ is a lattice $(0,1)$-homomorphism associated with a continuous order preserving mapping $f:\left(P^{\prime}, \tau^{\prime}\right) \rightarrow(P, \tau)$, then $h(x)=x^{\prime}$ if and only if $f^{-1}(X)=X^{\prime}$, where $X, X^{\prime}$ are the clopen decreasing sets representing $x, x^{\prime}$, respectively. It is easy to see that $h$ is one-to-one if and only if $f$ is onto, and that $h$ is onto if and only if $f$ is a one-to-one order embedding.

For a poset $P$, let $\operatorname{Min}(P)$ denote the set of all minimal elements of $P$. For any $Q \subseteq P$ let $(Q]=\{x \in P: x \leq q$ for some $q \in Q\}$, and $[Q)=\{y \in P: q \leq y$ for some $q \in Q\}$; define also $\operatorname{Min}(Q)=(Q] \cap \operatorname{Min}(P)$. Should $Q=\{x\}$, the sets $(Q],[Q), \operatorname{Min}(Q)$ will be abbreviated as $(x],[x), \operatorname{Min}(x)$, respectively. If the partial ordering $P$ is to be emphasized, $\operatorname{Min}(Q)$ will be written as $\operatorname{Min}_{P}(Q)$.

Since every prime ideal of a distributive lattice $L$ contains a minimal prime ideal, in the space $(P, \tau)$ representing $L$ the set $\operatorname{Min}(x)$ must be nonempty for every $x \in P$. Such a space has the p-property if $[Q)$ is a clopen set for every clopen decreasing subset $Q$ of $(P, \tau)$. Since $[Q)=[\operatorname{Min}(Q))$ for any decreasing $Q \subseteq P$, the set $P \backslash(Q)$ is easily seen to represent the pseudocomplement $x^{*}$ of the element $x$ in $L$ corresponding to the clopen decreasing set $Q$. Any compact totally order disconnected space $(P, \tau)$ satisfying these properties will henceforth be called a $p$ space. In view of $[\mathbf{3 3}]$ we may also conclude that, for $n>0$, a $p$-space $(P, \tau)$ is dual to a lattice in $B_{n}$ if and only if $|\operatorname{Min}(x)| \leq n$ for every $x \in P$, and that Boolean algebras are dual to those $p$-spaces for which $P=\operatorname{Min}(P)$. An order preserving mapping $f: P \rightarrow P^{\prime}$ is a $p$-map if $f(\operatorname{Min}(x))=\operatorname{Min}(f(x))$ for every $x \in P$. Any continuous $p$-map will be called a $p$-morphism.

As observed in [1] and H. A. Priestley [41], pseudocomplemented distributive lattices and $p$-spaces correspond under the duality of Theorem 2.2 as follows. 
THEOREM 2.3. $B_{\omega}$ is dually isomorphic to the category $T^{p}$ of all p-morphisms of $p$-spaces. For $n>0$, the restriction of this duality to $B_{n}$ gives the full subcategory $T_{n}^{p}$ of $T^{p}$ determined by all $p$-spaces $(P, \tau)$ satisfying $|\operatorname{Min}(x)| \leq n$ for all $x \in P$.

Finally, $(Q, \sigma)$ is a subspace of a $p$-space $(P, \tau)$ if it inherits the topology and the order of $(P, \tau)$ and if $\operatorname{Min}_{P}(Q) \subseteq Q$.

3. The varieties $B_{0}, B_{1}, B_{2}$. Throughout this section we will abuse the notation by writing $(P, \tau) \in V$ whenever $V$ is a variety of pseudocomplemented distributive lattices containing the algebra represented by the $p$-space $(P, \tau)$. A p-morphism is any continuous order preserving $p$-map.

For $i=3, \ldots, 7$ let the spaces $P_{i}$ of Figure 3.1 denote the corresponding pseudocomplemented distributive lattices $L_{i}$ of cardinality $i$ as shown in Figure 3.2.

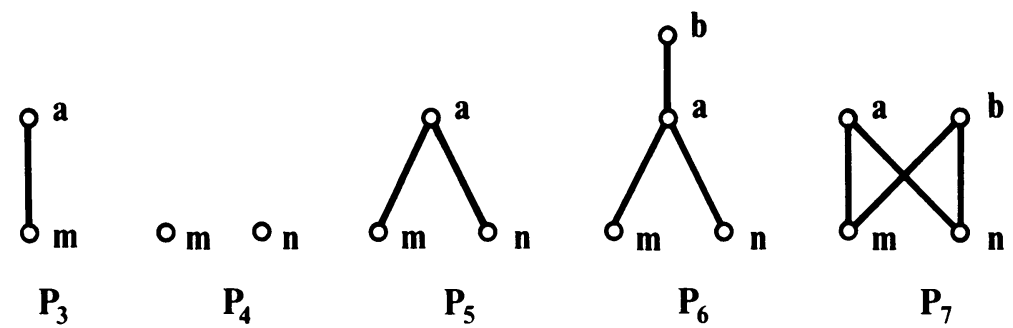

FIGURE 3.1

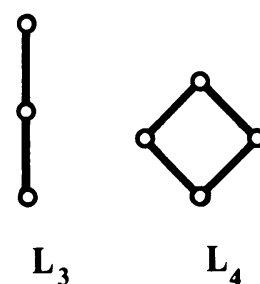

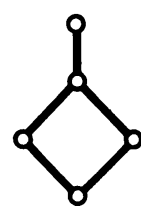

$\mathbf{L}_{5}$

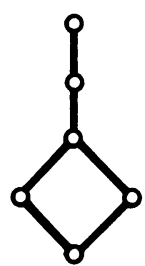

$\mathbf{L}_{6}$

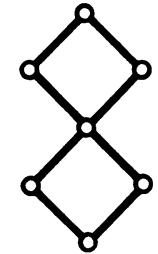

$\mathbf{L}_{7}$

FIGURE 3.2

We begin with another characterization of $p$-spaces, as given in Lemma 3.2.

LEMMA 3.1. Let $(P, \tau)$ be a compact totally order disconnected space with compact $\operatorname{Min}(P)$. Then for every set $K$ clopen in $\operatorname{Min}(P)$ there is a clopen decreasing $C \subseteq P$ with $C \cap \operatorname{Min}(P)=K$.

Proof. Let $K \subseteq \operatorname{Min}(P)$ be clopen, so that both $K$ and $L=\operatorname{Min}(P) \backslash K$ are compact. Fix $l \in L$; for every $k \in K$ there is a clopen decreasing $A_{k}$ containing $k$ and missing $l$. Hence $K \subseteq \bigcup A_{k}$ and the compactness implies that $K$ is contained in a clopen decreasing set $B_{l}$ such that $l \in P \backslash B_{l}$. Now the compact set $L$ is covered by the latter increasing clopen sets, and the compactness of $L$ shows that $L$ is contained in a clopen increasing set $D$ disjoint with $K$. The set $C=P \backslash D$ is decreasing and $C \cap \operatorname{Min}(P)=K$ as required.

LEMMA 3.2. Let $(P, \tau)$ be a compact totally order disconnected space such that $\operatorname{Min}(P)$ is compact. Then $(P, \tau)$ is a p-space if and only if every continuous $f: \operatorname{Min}(P) \rightarrow P_{4}$ can be extended to a p-morphism $g:(P, \tau) \rightarrow P_{5}$. 
Proof. Assume $(P, \tau)$ to be a $p$-space. Then $\operatorname{Min}(P)$ is a compact totally disconnected space. If $f: \operatorname{Min}(P) \rightarrow P_{4}$ is continuous, then $f^{-1}\{m\}$ is a clopen subset of $\operatorname{Min}(P)$, so that Lemma 3.1 implies the existence of a clopen decreasing $C \subseteq P$ with $f^{-1}\{m\}=C \cap \operatorname{Min}(P)$. The complement $D$ of $C$ is a clopen increasing set with $D \cap \operatorname{Min}(P)=f^{-1}\{n\}$. A clopen increasing $E \subseteq P$ with $E \cap \operatorname{Min}(P)=$ $f^{-1}\{m\}$ is found analogously. Extend $f$ to $g$ by setting $g(x)=a$ for $a \in D \cap E$, $m$ for $x \in E \backslash D$, and $n$ for $x \in D \backslash E$; it is easily seen that $g$ is a continuous order preserving $p$-map.

Conversely, assume the extension property; to show that $(P, \tau)$ is a $p$-space it suffices to establish the $p$-property. Let $S \subseteq P$ be a clopen decreasing set. Then $\operatorname{Min}(S)=\operatorname{Min}(P) \cap S$ is a clopen subset of $\operatorname{Min}(P)$, so that there exists a continuous $\operatorname{map} f: \operatorname{Min}(P) \rightarrow P_{4}$ with $f^{-1}\{m\}=\operatorname{Min}(S)$ which, by hypothesis, extends to a $p$-morphism $g:(P, \tau) \rightarrow P_{5}$. Clearly $[S)=[\operatorname{Min}(S))=g^{-1}\{m, a\}$, so that $[S)$ is clopen by continuity of $g$.

LEMMA 3.3. Let $x \not y$ in $(P, \tau) \in B_{\omega}$. Then there exists a $p$-morphism $g: P \rightarrow$ $P_{3}$ with $g(x) \not \leq g(y)$ if and only if $x \in P \backslash \operatorname{Min}(P)$.

Proof. Assume that $x \in P \backslash \operatorname{Min}(P)$. For every minimal $n \in P$ there exists a clopen decreasing set $Q_{n}$ containing $n$ and such that $x \notin P \backslash Q_{n}$. The union of these sets contains the closed subset $\operatorname{Min}(P)$ of the compact space $(P, \tau)$; finitely many of these sets then cover $\operatorname{Min}(P)$ and their union $R$ is a clopen decreasing set not containing $x$. There is also a clopen decreasing $Q$ containing $y$ but not $x$. Let $g^{-1}\{m\}=Q \cup R$ for the minimal element $m$ of $P_{3}$ and $g^{-1}\{a\}=P \backslash(Q \cup R)$; it is easy to see that $g$ is a $p$-morphism as required. Since $g(\operatorname{Min}(P))=\{m\}$ for any $p$-map $g, g(x)=a$ is possible only if $x \notin \operatorname{Min}(P)$.

Thus nonminimal elements can be separated by $p$-morphisms into $P_{3}$.

Lemma 3.4. Let $x \in \operatorname{Min}(P)$ and $x \not \leq y$ in $(P, \tau) \in B_{\omega}$. Then there exists a p-morphism $g: P \rightarrow P_{5}$ such that $\{g(x), g(y)\}=\operatorname{Min}\left(P_{5}\right)$. If $(P, \tau) \in B_{1}$ then $g(P) \subseteq P_{4}$ for any such $p$-map.

Proof. Since $x$ is minimal, $y \not \leq x$ as well, and there is a clopen decreasing set $Q$ such that $y \in Q$ and $x \in \operatorname{Min}(P) \backslash Q$. The set $Q \cap \operatorname{Min}(P)$ is clopen in $\operatorname{Min}(P)$, so that there is a continuous $f: \operatorname{Min}(P) \rightarrow P_{4}$ with $f^{-1}\{n\}=\operatorname{Min}(Q) \supseteq \operatorname{Min}(y)$. The extending $p$-morphism $g:(P, \tau) \rightarrow P_{5}$ must therefore satisfy $g(y)=n \neq g(x) \in$ $\operatorname{Min}\left(P_{5}\right)$.

This shows that minimal elements can be separated by $p$-morphisms into $P_{5}$ or, in the case of $B_{1}$, by $p$-morphisms into $P_{4}$.

COROLlaRY 3.5. If $(P, \tau) \in B_{\omega}$, then $g(x) \leq g(y)$ for every p-morphism $g: P \rightarrow P_{5}$ if and only if $\operatorname{Min}(x) \subseteq \operatorname{Min}(y)$.

ProOF. If $g: P \rightarrow P_{5}$ is a $p$-morphism with $g(x) \not \leq g(y)$ then $g(y)$ is a minimal element of $P_{5}$, so that $g(\operatorname{Min}(y))=\{g(y)\}$; since $g$ is a $p$-map, there must exist $t \in \operatorname{Min}(x) \backslash \operatorname{Min}(y)$. Conversely, for any such $t$ there is a $p$-morphism $h: P \rightarrow P_{5}$ with $\{h(t), h(y)\}=\operatorname{Min}\left(P_{5}\right)$ by Lemma 3.4 , and $h(x) \not L h(y)$ follows.

In view of this claim, some other means are needed to separate elements with the same nontrivial set of minimals in the absence of the Stone space $P_{3}$. 
LEMMA 3.6. Let $y, z \in(P, \tau) \in B_{\omega}$ be such that $\operatorname{Min}(y)=\operatorname{Min}(z)=M$ has more than one element. Then $z \notin y$ if and only if there exists a p-morphism $g: P \rightarrow P_{6}$ such that $g(y)=a$ and $g(z)=b$.

ProOF. By hypothesis, the element $z$ is not minimal; Lemma 3.3 gives a $p$ morphism $g_{3}: P \rightarrow P_{3}$ with $g_{3}(z)=a$ and $g_{3}(M \cup\{y\})=\operatorname{Min}\left(P_{3}\right)=\{m\}$. Furthermore, by Lemma 3.4 there is a $p$-morphism $g_{5}: P \rightarrow P_{5}$ with $g_{5}(M)=\operatorname{Min}\left(P_{5}\right)$ and, hence, $g(y)=g(z) \notin \operatorname{Min}\left(P_{5}\right)$.

Define $g: P \rightarrow P_{6}$ by $g(x)=b$ whenever $g_{3}(x)=a$ and $g(x)=g_{5}(x)$ for all other $x \in P$. It is easy to see that $g$ is a $p$-morphism with $g(y)=a<b=g(z)$. The converse implication is trivial.

COROLlARY 3.7. Let $(P, \tau) \in B_{2}$ be such that distinct $y, z \in P$ are incomparable whenever $\operatorname{Min}(y)=\operatorname{Min}(z)=M$ has more than one element. Then there exists a p-morphism $g: P \rightarrow P_{7}$ such that $g(y)=a$ and $g(z)=b$ for any such pair $\{y, z\}$. $P_{7}$.

ProOF. By hypothesis, the mapping $g$ of Lemma 3.6 is also a $p$-morphism to

LEMMA 3.8. If $i \in\{3,5,6\}$ and $Q \cong P_{i}$ is a subspace of $(P, \tau) \in B_{\omega}$, then there exists an idempotent $f \in \operatorname{End}(P, \tau)$ such that $f(P)=Q$. The claim also holds true for $P_{4}$, provided $(P, \tau) \in B_{1}$.

Proof. In all cases the lemma follows from the preceding separation statements. Thus, for instance, Lemma 3.4 implies that for every subspace $Q$ of $(P, \tau)$ isomorphic to $P_{5}$ there exists a $p$-morphism $g:(P, \tau) \rightarrow P_{5}$ with $g(Q)=P_{5}$; the composite $f=h g$ in which $h$ is the $p$-isomorphism of $P_{5}$ onto $Q \subseteq(P, \tau)$ is the required idempotent endormophism.

The following is similarly derived from Corollary 3.7.

LEMMA 3.9. If $Q \cong P_{7}$ is a subspace of $(P, \tau) \in B_{2}$ and if $(P, \tau)$ contains no subspace isomorphic to $P_{6}$, then there exists an idempotent $f \in \operatorname{End}(P, \tau)$ such that $f(P)=Q$.

Let $c_{m}$ denote the constant endomorphism of $(P, \tau) \in B_{\omega}$ with value $m \in$ $\operatorname{Min}(P)$.

LEMMA 3.10. Let $(P, \tau) \in B_{\omega}$ and let $E=\operatorname{End}(P, \tau)$. Then

(i) $f c_{m}=c_{f(m)}$ for all $f \in E$ and $m \in \operatorname{Min}(P)$,

(ii) $f \in E$ is a left zero of $E$ if and only if $f=c_{m}$ for some constant with $m \in \operatorname{Min}(P)$,

(iii) for $f \in E$ and $m \in \operatorname{Min}(P), m \in f(P)$ if and only if $c_{m} \in f E$.

Proof. (i) is clear. To see (ii) observe that, for $f \in E$ and $x \in P, c_{m} f(x)=$ $m=c_{m}(x)$ and that if $f$ is a left zero then, for $m \in \operatorname{Min}(P), f=f c_{m}=c_{f(m)}$. Consider (iii). If $m \in f(P)$, then $m=f(n)$ for some $n \in \operatorname{Min}(P)$. Since $f c_{n}(x)=m$ for all $x \in P, c_{m}=f c_{n}$ follows. Conversely, $c_{m}=f g$ implies $m=c_{m}(x)=f g(x)$ for any $x \in P$. The proof is complete.

Notation. For idempotent $f, g \in E=\operatorname{End}(P, \tau) \in B_{\omega}$ and a $p$-morphism $\alpha: f(P) \rightarrow g(P)$, the composite $F(\alpha)=g \alpha f$ is an endomorphism of $(P, \tau)$. Following are some properties of $F$. 
LEMMA 3.11. If $f, g, \alpha$ and $F$ are as above and if $\beta: g(P) \rightarrow h(P)$ for some idempotent $h \in E$, then, for $H(f, g)=\operatorname{Hom}(f(P), g(P))$,

(i) $F(\beta \alpha)=F(\beta) F(\alpha)$,

(ii) $F(H(f, g))=g E \cap E f=\{e \in E: g e f=e\}$,

(iii) $F(e \uparrow f(P))=e$ for every $e \in g E \cap E f$,

(iv) $F$ is one-to-one on each $H(f, g)$.

In particular, $F$ is a constant-preserving semigroup isomorphism of $\operatorname{End}(f(P))$ onto $f E \cap E f \subseteq \operatorname{End}(P)$.

ProOF. To see (i) note that $F(\beta) F(\alpha)=h \beta g g \alpha f=h \beta g \alpha f=h \beta \alpha f=F(\beta \alpha)$. Next observe that $e=g e_{1}=e_{2} f$ implies $g e f=g^{2} e_{1} f=g e_{1} f=e_{2} f^{2}=e_{2} f=e$; hence $g e f=e$ is equivalent to $e \in g E \cap E f$. To see that $F(\alpha) \in g E \cap E f$ it is enough to recall that $f, g$ are idempotent; since $e(f(P)) \subseteq g(P)$ for every $e \in g E \cap E f$, (ii) follows immediately. (iii) and (iv) are also easily obtained.

LEMMA 3.12. For $(P, \tau) \in B_{\omega}$, if $\operatorname{End}(P, \tau) \cong \operatorname{End}\left(P_{i}\right)$ for $i=3,5,6,7$, then $(P, \tau) \cong P_{3}, P_{4}$ or $P_{5}, P_{6}, P_{7}$, respectively.

Proof. $\operatorname{End}\left(P_{3}\right)=\left\{1, c_{m}\right\}$, so that $\operatorname{Min}(P, \tau)$ is a singleton by Lemma 3.10 (ii); should $P \backslash \operatorname{Min}(P)$ have more than one element, Lemma 3.3 would imply the existence of at least two idempotent endomorphisms distinct from 1 and from $c_{m}$.

Again by Lemma $3.10(\mathrm{ii}), \operatorname{Min}(P, \tau)=\{m, n\}$ in all remaining cases. If there is an element $x \in P \backslash \operatorname{Min}(P)$ with $\operatorname{Min}(x)=\{m\}$, then Lemma 3.3 implies the existence of a nonconstant idempotent $f$ with $f c_{m}=f c_{n}=c_{m}$. Since every $z \in$ $P_{i} \backslash \operatorname{Min}\left(P_{i}\right)$ satisfies $\operatorname{Min}(z)=\operatorname{Min}\left(P_{i}\right)$ for $i=4, \ldots, 7$, there is no such idempotent in any of the corresponding $\operatorname{End}\left(P_{i}\right)$. We see that $\operatorname{Min}(x)=\operatorname{Min}(P, \tau)$ for any $x \in P \backslash \operatorname{Min}(P)$.

Let $F$ be the set of all $f \in \operatorname{End}(P, \tau)$ with $f c_{m}=c_{m}$ and $f c_{n}=c_{n}$. By Lemma 3.8, for every $a \in P \backslash \operatorname{Min}(P)$ there exists an idempotent $e_{a} \in F$ with $e_{a}(P)=$ $\{m, n, a\}$. Since these idempotents are exactly the left zeros of the semigroup $F$, the space $(P, \tau)$ is isomorphic to $P_{4}$ or to $P_{5}$ if and only if $F$ is trivial. Otherwise, $P \backslash \operatorname{Min}(P)$ has exactly two elements; these elements are incomparable just when there exists a nontrivial $f \in F$ such that $f^{2}$ is the unit of $F$, and this property distinguishes $P_{7}$ from $P_{6}$. The proof is complete.

A $p$-space $(P, \tau) \in B_{2}$ lies in $B_{1}$ if and only if it does not contain a subspace isomorphic to $P_{5}$; the following is obtained immediately from 3.8, 3.11 and 3.12.

PROPOSITION 3.13. The endomorphism monoid of a $p$-space $(P, \tau) \in B_{2}$ determines whether or not each of $P_{3}, P_{4}$ or $P_{5}, P_{6}$ is a subspace of $(P, \tau)$.

Similarly, the following is seen to hold by 3.9 .

PROPOSITION 3.14. If $(P, \tau) \in B_{2}$ does not contain a subspace isomorphic to $P_{6}$, then $\operatorname{End}(P, \tau)$ determines whether or not there is a subspace of $(P, \tau)$ isomorphic to $P_{7}$.

The lemma below provides a first step needed to recover the structure of a space in $B_{2}$ from its endomorphism monoid.

LEMMA 3.15. Let $(P, \tau),(Q, \sigma) \in B_{2}$, and let $\Phi$ be an isomorphism of $\operatorname{End}(P, \tau)$ onto $\operatorname{End}(Q, \sigma)$. Then there is a homeomorphism $\varphi$ of the Boolean subspace $\operatorname{Min}(P)$ 
onto the subspace $\operatorname{Min}(Q)$ of $Q$ such that $\Phi(f)(\varphi(m))=\varphi(f(m))$ for all $f \in$ $\operatorname{End}(P, \tau)$ and $m \in \operatorname{Min}(P)$. In particular, $m \in f(P)$ is equivalent to $\varphi(m) \in$ $\Phi(f)(Q)$ for every $m \in \operatorname{Min}(P)$.

PrOOF. The isomorphism $\Phi$ establishes a bijection between the sets of left zeros of the respective endomorphism monoids; by Lemma 3.10 there is a bijection $\varphi$ of $\operatorname{Min}(P)$ onto $\operatorname{Min}(Q)$ defined by $\Phi\left(c_{m}\right)=c_{\varphi(m)}$. Furthermore, for any $f \in$ $\operatorname{End}(P, \tau)$ and any $m \in \operatorname{Min}(P)$, we see that, for all $q \in Q$,

$$
\begin{aligned}
\Phi(f)(\varphi(m)) & =\Phi(f)\left(c_{\varphi(m)}(q)\right)=\Phi(f) \Phi\left(c_{m}\right)(q) \\
& =\Phi\left(f c_{m}\right)(q)=\Phi\left(c_{f(m)}\right)(q)=c_{\varphi f(m)}(q)=\varphi(f(m)) .
\end{aligned}
$$

To show that $\varphi$ is a homeomorphism, let $A \subseteq \operatorname{Min}(P)$ be a clopen set. There is a $p$-morphism $f: \operatorname{Min}(P) \rightarrow P_{4}$ with $f^{-1}\{a\}=A$, so that, by Lemma 3.2 , there is a $p$-morphism $g:(P, \tau) \rightarrow P_{5}$ extending $f$. Whether $g(P) \cong P_{5}$ or $g(P) \cong P_{4}$ there exists an idempotent endomorphism $h$ of $(P, \tau)$ such that $\operatorname{Min}(P) \cap h^{-1}\{a\}=A$, where $\{a\}=h(A)$. Since $\varphi$ is a bijection of $\operatorname{Min}(P)$ onto $\operatorname{Min}(Q)$, for every $m \in$ $\operatorname{Min}(P), \Phi(h)(\varphi(m))=\varphi(h(m))=\varphi(a)$ is equivalent to $\varphi(m) \in \varphi(A)$. Therefore $\varphi(A)=\operatorname{Min}(Q) \cap \Phi(h)^{-1}\{\varphi(a)\}$ is a clopen subset of $\operatorname{Min}(Q)$. Since the Boolean spaces $\operatorname{Min}(P), \operatorname{Min}(Q)$ have clopen bases and are compact, $\varphi$ is a homeomorphism.

DEFINITION. A subset $S$ of $\operatorname{End}(P, \tau)$ is called a separation set if

(i) $s(P)$ is finite for each $s \in S$,

(ii) if $x \not y$, then $s(x) \not \leq s(y)$ for some $s \in S$.

It is an order separation set if, in (ii), it is also the case that $s(y) \in \operatorname{Min}(P)$.

The role of (order) separation sets is apparent from the claim below.

LEMMA 3.16. Let $(P, \tau),(Q, \sigma) \in B_{\omega}$ have the same underlying set $X$ and let $S \subseteq \operatorname{End}(P, \tau) \cap \operatorname{End}(Q, \sigma)$. Then

(i) if $S$ is a separation set for $(P, \tau)$ then $\tau=\sigma$,

(ii) if $S$ is an order separation set for $(P, \tau)$, then $\tau=\sigma$ and $P=Q$.

ProOF. If $S$ is a separation set for $(P, \tau)$, then conditions (i) and (ii) from the definition imply that sets of the form $s^{-1}\{s(x)\}$ with $s \in S$ and $x \in X$ form a clopen subbase in either compact Hausdorff topology; this is possible only if $\tau=\sigma$.

In view of Lemma 3.15 we may assume that $\operatorname{Min}(P)=\operatorname{Min}(Q)$. Let $S \subseteq$ $\operatorname{End}(P, \tau) \cap \operatorname{End}(Q, \sigma)$ be order separation. If $x \not \leq y$ in $P$, then $s(x) \not \leq s(y) \in \operatorname{Min}(P)$ for some $s \in S$, while $x \leq y$ in $Q$ would yield $s(x) \leq s(y) \in \operatorname{Min}(Q)$ in $Q$ and hence $s(x)=s(y)$. This concludes the proof of (ii) since $S$ is also a separation set.

Following is the first instance in which the underlying set can be recovered from the endomorphism monoid.

Proposition 3.17. Let $(P, \tau),(Q, \sigma) \in B_{2}$ and let $\Phi$ be an isomorphism of $\operatorname{End}(P, \tau)$ onto $\operatorname{End}(Q, \sigma)$. If both spaces contain a subspace isomorphic to $P_{5}$, then there is a bijection $\psi$ of $P$ onto $Q$ extending the homeomorphism $\varphi$ of $\operatorname{Min}(P)$ onto $\operatorname{Min}(Q)$ from 3.15 and satisfying $\Phi(f) \circ \psi=\psi \circ f$ for any $f \in \operatorname{End}(P, \tau)$. Furthermore, for $m \in \operatorname{Min}(P)$ and $x \in P, m \leq x$ if and only if $\psi(m) \leq \psi(x)$.

ProOF. Let $P_{5} \cong\{m, n, a\} \subseteq(P, \tau)$ and $P_{5} \cong\{r, s, b\} \subseteq(Q, \sigma)$ with $a, b$ nonminimal. Without a loss of generality we may assume that a Boolean space $(M, \rho)$ is the subspace of minimals of either $p$-space. Therefore Lemma 3.15 translates to 
say that, for every $f \in \operatorname{End}(P, \tau)$, the restrictions of $f$ and of $\Phi(f)$ coincide on $(M, \rho)$.

There exists an idempotent $f \in \operatorname{End}(P, \tau)$ for which $f(r)=m$ and $f(s)=n$; this follows from Lemma 3.2 since $(M, \rho)$ is a Boolean space. Thus $\Phi(f)(r)=$ $m, \Phi(f)(s)=n$ as well and, therefore, $\{m, n, \Phi(f)(b)\}$ is a subspace of $(Q, \sigma)$ isomorphic to $P_{5}$. We see that $P(m, n)=\left\{x \in P: \operatorname{Min}_{P}(x)=\{m, n\}\right\}$ is nonempty if and only if $Q(m, n)$, defined similarly in $(Q, \sigma)$, is nonempty.

Select and fix an idempotent $f \in \operatorname{End}(P, \tau)$ with $f(P)=\{m, n, a\} \cong P_{5}$. The endomorphism $\Phi(f)$ of $(Q, \sigma)$ is idempotent and its image is isomorphic to $P_{4}$ or to $P_{5}$ by Lemmas 3.11 and 3.12. Since $Q(m, n)$ is nonempty, the first case cannot occur; let $b$ denote the nonminimal element of $\Phi(f)(Q)$. For every $x \in P(r, s)$ there also is an idempotent $g_{x}$ such that $g_{x}(P)=\{r, s, x\}$; by Lemma 3.11, $e=$ $g_{x} e f$ for exactly two nonconstant $e \in \operatorname{End}(P, \tau)$, and $e(a)=x$ for both of these. Thus the two maps $\Phi(e)=\Phi\left(g_{x}\right) \Phi(e) \Phi(f)$ also are nonconstant; since $\Phi\left(g_{x}\right)(Q)$ is isomorphic to a subspace of $P_{5}$ as well and $\Phi(f)(Q) \cong P_{5}$, there is a uniquely determined $\psi(x)=\Phi(e)(b)$ in $Q(r, s)$. For $l \in M, l<x$ if and only if $l \in e(P)$. By Lemma 3.10(iii), this is equivalent to $c_{l} \in e E_{P}$, where $E_{P}=\operatorname{End}(P, \tau)$; that is, $c_{l}=\Phi\left(c_{l}\right) \in \Phi(e) \Phi\left(E_{P}\right)=\Phi(e) E_{Q}$ or, equivalently, $l \in \Phi(e)(Q)$. Thus, $m<x$ is equivalent to $m<\psi(x)$ for any $x$ with $|\operatorname{Min}(x)|>1$. For $x \in P \backslash M$ with $\operatorname{Min}(x)=\{r\}$ let $g_{x}(P)=\{r, x\}$ for an idempotent endomorphism $g_{x}$; there is a unique nonconstant $g_{x} e f=e$ with $e(a)=x$, so that $\psi(x)=\Phi(e)(b)$ is well defined and $\psi(x) \in P \backslash M$. Similarly, $l<x$ if and only if $l<\psi(x)$ for $l \in M$. The definition of $\psi$ can now be summarized as follows. For every $x \in P$ there is an endomorphism $e_{x}=g_{x} e_{x} f$ of $(P, \tau)$ such that $e_{x}(a)=x ; \psi(x)$ is then defined as $\Phi\left(e_{x}\right)(b)$ for any such endomorphism. Clearly then $m \leq x$ if and only if $m \leq \psi(x)$ for any $m \in M$. For every $h \in \operatorname{End}(P, \tau)$ we thus have $h e_{x}(a)=h(x)$ and, therefore,

$$
\begin{aligned}
\Phi(h)(\psi(x)) & =\Phi(h) \Phi\left(e_{x}\right)(b)=\Phi\left(h e_{x}\right)(b) \\
& =\Phi\left(e_{h(x)}\right)(b)=\psi(h(x)) .
\end{aligned}
$$

It is obvious that $\psi$ is one-to-one. To see that $\psi$ is onto, note that the argument may be reversed: for every $y \in Q$ there is an endomorphism $k_{y}=k_{y} \Phi(f)$ such that $k_{y}(b)=y$. Thus $\Phi^{-1}\left(k_{y}\right)(a)$ is the element whose $\psi$-image is $y$. The proof is complete.

THEOREM 3.18. Algebras in $B_{1}$ are uniquely determined by their endomorphism monoids.

ProOF. If $(P, \tau),(Q, \sigma) \in B_{1}$ have isomorphic endomorphism monoids then Proposition 3.13 implies that either they are both Boolean and hence isomorphic by Lemma 3.15, or, by Proposition 3.13, both contain $P_{3}$ as a subspace. Assume again that the spaces share the subspace of their respective minimals. If $\{m, a\} \subseteq(P, \tau)$, then there exists a nonconstant idempotent endomorphism $f$ with $f(P)=\{m, a\}$; for every $x \in P \backslash M$ there is an endomorphism $e_{x}=e_{x} f$ with $e_{x}(a)=x$. Similarly to the proof of 3.13 we establish a bijection $\psi$ of $P$ onto $Q$ such that $\Phi(h) \circ \psi=\psi \circ h$ for any endomorphism $h$ of $(P, \tau)$. The underlying sets of $P$ and $Q$ may thus be identified. Then 3.3 and 3.4 combine to show that the endomorphisms whose images are isomorphic to $P_{3}$ or to $P_{4}$ form an order separation set, so that the spaces coincide by 3.16 . 
THEOREM 3.19. If $(P, \tau),(Q, \sigma) \in B_{2}$ have isomorphic endomorphism monoids and if $P_{3}$ is isomorphic to a subspace of one of them, then the spaces must be isomorphic.

ProOF. Recall that under the hypothesis both spaces must contain a copy of $P_{3}$. If $P_{5}$ is not a subspace of either, Theorem 3.18 applies. Hence assume that $(P, \tau)$ contains both $P_{3}$ and $P_{5}$ as subspaces. If $f, g$ are idempotent endomorphisms such that $f(P) \cong P_{5}$ and $g(P) \cong P_{3}$, then, by 3.11 , there is a nonconstant $e=g e f$ in $\operatorname{End}(P, \tau)$. From 3.11 and 3.12 we see that $\Phi(g)(Q) \cong P_{3}$ and $\Phi(f)(Q) \cong P_{4}$ or $P_{5}$. Should the first case occur, the set of all $h \in \operatorname{End}(Q, \sigma)$ with $h=\Phi(g) h \Phi(f)$ will consist of a single constant. Thus $\Phi(Q) \cong P_{5}$ and 3.17 implies that the underlying sets of $P$ and $Q$ and also their endomorphism monoids can be identified. By 3.3 and 3.4 , the endomorphisms with images contained in $P_{3}$ or $P_{5}$ form an order separating set, so that $(P, \tau)$ and $(Q, \sigma)$ are isomorphic by 3.12. This completes the proof.

From now on we will assume that $P_{3}$ is a subspace of neither space. The first case to consider will be that of $P_{5}$ being contained in both. Therefore, by 3.17, we may assume that the two spaces share the space $M$ of minimals, have the same underlying set $X$, and $\operatorname{End}(P, \tau)=\operatorname{End}(Q, \sigma)$.

If $P \backslash M$ contains a comparable pair, say $a<b$, then $\operatorname{Min}(b)=\operatorname{Min}(a)=\{m, n\}$ and $\{m, n, a, b\} \cong P_{6}$. Thus, by 3.11 and 3.12 , we obtain that $\{m, n, a, b\}$ is a subspace of $(Q, \sigma)$ isomorphic to $P_{6}$; note, however, that possibly $a>b$ in $Q$. Select an idempotent $h \in \operatorname{End}(P, \tau)$ with $h(P)=\{m, n, a, b\} \cong P_{6}$; see Lemma 3.6. The sets $P(r, s)=Q(r, s)$ are pairwise disjoint and cover $X \backslash M$, so that $x \leq y$ in $P$ iff $x \leq y$ in $Q$ unless $x, y \in P(r, s)$ for some $\{r, s\} \subseteq M$, by Proposition 3.17. For every pair $x<y$ in $P(r, s)$ the subspace $\{r, s, x, y\}$ is isomorphic to $P_{6}$; hence there is an endomorphism $g$ with $g(a)=x$ and $g(b)=y$. Thus $x<y$ or $y<x$ in $Q$ as long as $a<b$ or $b<a$ holds, respectively, in $Q$. In either case from 3.4, 3.5, 3.6, 3.12 it follows that $\sigma=\tau$ since the endomorphisms into $P_{6}$ form a separation set. If $a<b$ in $Q$, then $Q=P$. If $b<a$ in $Q$, then the $Q$-order of each $P(r, s)$ is dual to the $P$-order of $P(r, s)$; that is, the identity map of $X \backslash M$ is a dual isomorphism of $P \backslash M$ onto $Q \backslash M$. We see that there possibly is another space $(Q, \tau)$ with $\operatorname{End}(P, \tau)$ as its endomorphism monoid.

To see that this is indeed the case, let $(Q, \tau)$ be obtained from the $p$-space $(P, \tau)$ by the reversal of the partial order of $P \backslash M$. We need to show that $(Q, \tau)$ is a $p$-space whose endomorphism monoid coincides with that of $(P, \tau)$.

To verify that $\operatorname{End}(P, \tau) \subseteq \operatorname{End}(Q, \tau)$, note that the topologies coincide, and that $\operatorname{Min}_{P}(x)=\operatorname{Min}_{Q}(x)$ for all $x \in X$. Let $f \in \operatorname{End}(P, \tau)$ and $x \leq y$ in $Q$. If $x \in M$ then $x \leq y$ in $P$ and, consequently, $f(x) \in M$ and $f(x) \leq f(y)$ in $P$; thus $f(x) \leq f(y)$ also in $Q$. If $x \in Q \backslash M$ then $x, y \in Q(r, s)=P(r, s)$ for some $\{r, s\} \subseteq M$, so that $x \geq y$ in $P$. If $f(x) \in M$ then $f(y)=f(x)$ since $f$ is order preserving; the same conclusion is drawn if $f(y) \in M$, for $f$ is a $p$-map and $P_{3}$ is not a subspace of $(P, \tau)$. Thus $f(x) \in X \backslash M$ iff $f(y) \in X \backslash M$ and hence in this case too $f(x) \leq f(y)$ in $Q$ by definition. We see that every $p$-endomorphism of $(P, \tau)$ is also a $p$-endomorphism of $(Q, \tau)$. The argument can be reversed to conclude that $\operatorname{End}(P, \tau)=\operatorname{End}(Q, \tau)$.

Clearly $(Q, \tau)$ is compact; to see that it is totally order disconnected, let $x \not$ $y$ in $Q$. Assume first that there is some $m$ in $\operatorname{Min}_{Q}(x) \backslash \operatorname{Min}_{Q}(y)$. Then $m \in$ $\operatorname{Min}_{P}(x) \backslash \operatorname{Min}_{P}(y)$, so that there is an endomorphism $g$ of $(P, \tau)$ with $g(X) \subseteq P_{5}$ 
and $\{g(y), g(m)\}=\operatorname{Min}\left(P_{5}\right)$ by Lemma 3.4. Since $g$ is also an endomorphism of $(Q, \tau)$, the set $g^{-1}\{g(y)\}$ is clopen, decreasing, and does not contain $x$. By symmetry, we may assume that $\operatorname{Min}_{Q}(x)=\operatorname{Min}_{Q}(y)$; since $(Q, \tau)$ does not contain a subspace isomorphic to $P_{3}$, it follows that $x, y \in Q(r, s)$. Hence $x \nsupseteq y$ in $(P, \tau)$ and there exists $f \in \operatorname{End}(P, \tau)$ with $f(X)=\{m, n, a, b\}$ such that $f(x)=a$ and $f(y)=b$. Since $a<b$ both lie in $P(f(r), f(s))=Q(f(r), f(s))$, the opposite $a>b$ holds in $Q$; thus $f^{-1}\{a\}$ is a clopen increasing set of $Q$ containing $x$ and missing $y$. As a result, $(Q, \tau)$ is totally order disconnected.

To show that $(Q, \tau)$ is a $p$-space it remains to show that every continuous map of $(M, \rho)$ into $P_{4}$ extends to a $p$-morphism of $(Q, \tau)$ into $P_{5}$. This, however, is trivial since $(P, \tau)$ has the $p$-property and every $Q(r, s)=P(r, s)$ will be collapsed under the extension. By Lemma 3.2, $(Q, \tau)$ is a $p$-space.

Let $\left(P^{+}, \tau\right)$ denote the space obtained from $(P, \tau)$ in this manner and let $L^{+}$ denote the algebra in $B_{2}$ that is represented by $\left(P^{+}, \tau\right)$ provided $L$ corresponds to $(P, \tau)$. It is clear that $L \cong L^{+}$if and only if $P \cong P^{+}$, that is, if and only if the poset $P(r, s)$ is self-dual for all $\{r, s\} \subseteq \operatorname{Min}(P)$. We have proved the following.

Proposition 3.20. For $(P, \tau) \in B_{2}$ such that $P_{3}$ is not a subspace of $P$ while $P_{6}$ is, there exists at most one other space $\left(P^{+}, \tau\right) \in B_{2}$ such that $\operatorname{End}(P, \tau) \cong$ $\operatorname{End}\left(P^{+}, \tau\right)$. The underlying sets of these two possible spaces coincide and $P^{+}$has $P_{6}$ amongst its subspaces but not $P_{3}$.

The next case to consider is that of a space $(P, \tau) \in B_{2}$ containing neither $P_{3}$ nor $P_{6}$ as a subspace; we shall still assume that $P_{5}$ is a subspace of both $(P, \tau)$ and $(Q, \sigma)$. Recall that $(Q, \sigma)$ does not contain $P_{3}$ or $P_{6}$ either, and that the underlying set and the order of the two spaces coincide since every $P(r, s)=Q(r, s)$ must be an antichain. If $P_{7} \cong\{m, n, a, b\} \subseteq(P, \tau)$, then $P_{7}$ is also contained in $(Q, \sigma)$ by 3.14. But then the endomorphisms into $P_{7}$ will form a separation set; see Corollary 3.7. Otherwise every $P(r, s)=Q(r, s)$ has at most one element and separation is obtained by mapping into $P_{5}$; see 3.4 . The following has been established.

THEOREM 3.21. If two spaces in $B_{2}$ with isomorphic endomorphism monoids contain $P_{5}$ and one of them does not contain $P_{6}$, then the other one does not contain $P_{6}$ and they are isomorphic.

The remaining case is that of $(P, \tau)$ containing no copy of either $P_{3}$ or $P_{5}$, while $(Q, \sigma)$ contains $P_{5}=\{m, n, a\}$, but, by Lemma 3.12 , not $P_{3}, P_{6}$, or $P_{7}$. By 3.15, $(P, \tau)$ is a Boolean space homeomorphic to $\operatorname{Min}(Q)$. For every pair $\{r, s\} \subseteq \operatorname{Min}(Q)$, the set $Q(r, s)$ has at most one element. For every pair $\{r, s\} \subseteq \operatorname{Min}(Q)$ there is, by 3.2, a $p$-endomorphism $f$ of $(Q, \sigma)$ such that $f(\{m, n\})=\{r, s\}$; thus every $Q(r, s)$ has exactly one element. By Theorem 3.21, there is, up to isomorphism, at most one such space; the existence of $(Q, \sigma)$ remains to be shown.

For $f: P \times P \rightarrow P^{+}=\{\{m, n\}: m, n \in P\}$ given by $f(m, n)=\{m, n\}$, let $\sigma$ denote the quotient topology on $P^{+}$; that is, $B \subseteq P^{+}$is open if and only if $f^{-1}(B)$ is open in the product space $(P, \tau)^{2}$. Let $P^{+}$be ordered by inclusion; clearly, $P^{+}(\{m\},\{n\})$ has exactly one element for every pair $m, n \in P$. Since $(P, \tau)^{2}$ is a Boolean space, $\left(P^{+}, \sigma\right)$ is compact. Observe that for any clopen $C \subseteq P$, $(m, n) \in A=(C \times P) \cup(P \times C)$ if and only if $\{m, n\} \in f(A)$. Consequently, $f^{-1}(f(A))=A$ and $f(A)$ is clopen. Clearly, $\operatorname{Min}\left(P^{+}\right) \cap f(A)=\{\{m\}: m \in C\}$, so that $P$ is homeomorphic to $\operatorname{Min}\left(P^{+}\right)$. Further, $f(A)$ is increasing since $(m, n) \in A$ 
for every $n \in P$ whenever $m \in C$. Thus, if $X \not \leq Y \in P^{+}$, then, for $m \in X \backslash Y$, $C$ may be chosen such that $m \in C$ and $C \cap Y=\varnothing$; consequently, $X \in f(A)$ and $Y \notin f(A)$. Thus $\left(P^{+}, \sigma\right)$ is totally order disconnected. If $D \subseteq P^{+}$is clopen decreasing, then $f^{-1}(D)=\bigcup\left(C_{i} \times C_{i}^{\prime}: i<k\right)$ where $C_{i}, C_{i}^{\prime} \subseteq P$ are clopen. Then $f(B)$ is clopen, where $B=\bigcup\left(\left(C_{i} \times P\right) \cup\left(P \times C_{i}\right): i<k\right)$. Since $D$ is decreasing, $(m, n) \in B$ if and only if $\{m\} \in B$ or $\{n\} \in B$ : thus $f(B)=[D)$, and $\left(P^{+}, \sigma\right)$ is a $p$-space.

Since, for any $p$-morphism $g$ of $\left(P^{+}, \sigma\right)$ to itself, $g\left(\operatorname{Min}\left(P^{+}\right)\right) \subseteq \operatorname{Min}\left(P^{+}\right)$, all that remains to be shown is that any continuous map $g$ of $(P, \tau)$ extends uniquely to a $p$ morphism of $\left(P^{+}, \sigma\right)$. Define $g^{+}\{x, y\}=\{g(x), g(y)\}$ for all $\{x, y\} \in P^{+}$; it is clear that $g^{+}$is the only $p$-map extending $g$ when $(P, \tau)$ is identified with $\operatorname{Min}\left(P^{+}\right)$. The map $g \times g: P \times P \rightarrow P \times P$ is continuous, and so is $f \circ(g \times g)=g^{+} \circ f$; to establish the continuity of $g^{+}$it is enough to recall that $f$ is a quotient map. Altogether, $\Phi$ defined by $\Phi(g)=g^{+}$is an isomorphism of $\operatorname{End}(P, \tau)$ onto $\operatorname{End}\left(P^{+}, \sigma\right)$. It is clear that these spaces are nonisomorphic.

THEOREM 3.22. For every Boolean space $(P, \tau)$ there is a unique space $\left(P^{+}, \sigma\right)$ in $B_{2}$ such that $\operatorname{End}(P, \tau) \cong \operatorname{End}\left(P^{+}, \sigma\right)$ and $(P, \tau) \not\left(P^{+}, \sigma\right)$; the space $\left(P^{+}, \sigma\right)$ lies in $B_{2} \backslash B_{1}$.

Following is a summary of the results of the present section in an algebraic form.

THEOREM 3.23. At most two nonisomorphic pseudocomplemented distributive lattices in $B_{2}$ may have isomorphic endomorphism monoids: either one of the pair is a Boolean algebra and the other is a unique algebra in $B_{2} \backslash B_{1}$, or both algebras of the pair lie in $B_{2} \backslash B_{1}$. In both cases, any quotient of either one of the algebras that lies in $B_{1}$ is Boolean.

4. The variety $B_{3}$. An undirected graph $(X, R)$ is a set $X$ together with a collection $R$ of two-element subsets of $X$. For graphs $\left(X_{1}, R_{1}\right),\left(X_{2}, R_{2}\right)$, a mapping $\varphi: X_{1} \rightarrow X_{2}$ is compatible iff, for $\{x, y\} \in R_{1},\{\varphi(x), \varphi(y)\} \in R_{2}$.

A variety $V$ of algebras is almost universal if the category of all compatible mappings of undirected graphs is isomorphic to the class of all nonconstant homomorphisms between algebras from a subclass $D$ of $V$.

The object of this section is to show that the variety $B_{3}$ is almost universal and, hence, by definition, that $B_{4}, \ldots, B_{\omega}$ are also almost universal. As observed in $\S 1$, there are a number of immediate consequences each of which illustrates the marked difference between the varieties $B_{2}$ and $B_{3}$. Using ideas from [1], G. Grätzer, H. Lakser and R. W. Quackenbush [20], and [38], it is not hard to show that, for any integer $n$, there exist an integer $N$ and a collection $\left(L_{i} \in B_{3}: i<n\right)$ of finite pseudocomplemented distributive lattices such that, for distinct $i, j<n, L_{i} \not L_{j}$ and $\operatorname{End}\left(L_{i}\right)$ consists of the identity and $N$ right zeros. This weaker result (which is one of the consequences of Theorem 1.1(iv)) already indicates the change at $B_{3}$. Since it is easily obtainable en route to the main construction, it will, by way of motivation, be derived shortly.

Some more notation is needed.

The height of a poset $P$ is the largest positive integer $n$ for which $P$ has a chain of cardinality $n$; if no such integer exists then the height of $P$ is infinite. A poset $P$ is a triple order if (i) it has height at most two, (ii) for $x \in P$, if $x \notin \operatorname{Min}(P)$ then $|\operatorname{Min}(x)|=3$, and (iii) for $x, y \in P, \operatorname{Min}(x)=\operatorname{Min}(y)$ implies $x=y$. 
For a graph $(X, R)$, the subgraph induced by $Y \subseteq X$ is the graph $(Y, S)$, where, for $x, y \in Y,\{x, y\} \in S$ iff $\{x, y\} \in R$. The complementary graph of $(X, R)$ is the graph $\left(X, R^{\prime}\right)$, where, for distinct $x, y \in X,\{x, y\} \in R^{\prime}$ iff $\{x, y\} \notin R$. A graph $(X, R)$ is connected if, for distinct $x, y \in X$, there exist an integer $n$ and, for $i \leq n$, $x_{i} \in X$ such that $x=x_{0}, y=x_{n}$, and, for $i<n,\left\{x_{i}, x_{i+1}\right\} \in R$. Finally, a graph is automorphism rigid if the only compatible bijection to itself with a compatible inverse is the identity. A graph is endomorphism rigid if the only compatible map to itself is the identity.

Choose a finite graph $(G, R)$ satisfying the following properties:

(i) $(G, R)$ is automorphism rigid;

(ii) $(G, R),\left(G, R^{\prime}\right)$ are connected;

(iii) $(G, R) \not\left(G, R^{\prime}\right)$;

(iv) $|G| \geq 12$;

(v) for $H \subseteq G$ such that $|H|+2 \geq|G|$, if $(H, S)$ denotes the induced subgraph of $(G, R)$, then $|S|,\left|S^{\prime}\right| \geq 5$.

Such graphs abound. For example, by Z. Hedrlín and A. Pultr [25] (see also A. Pultr and V. Trnková [43]), the graph of Figure 4.1 satisfies (i)-(v); it is, in fact, endomorphism rigid.

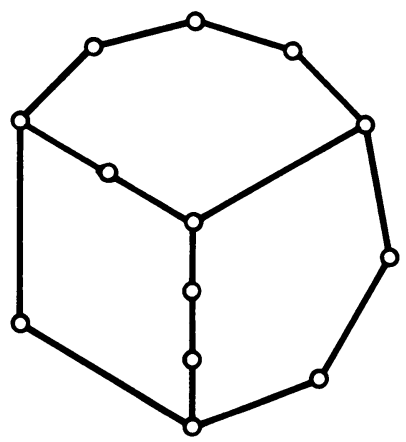

FIGURE 4.1

Let $T=G \cup R \cup R^{\prime} \cup\{a, b\}$, where $a, b$ are distinct elements. Define a partial order on $T$ by the following:

(i') for $r \in R, \operatorname{Min}(r)=\{a\} \cup r$;

(ii') for $r^{\prime} \in R^{\prime}, \operatorname{Min}\left(r^{\prime}\right)=\{b\} \cup r^{\prime}$.

Further, let $T^{\prime}=T \cup\{c, d, e, f\}$ for distinct elements $c, d, e$ and $f$ not contained in $T$. Choose, for $1 \leq i \leq 12$, distinct $g_{i} \in G$ (this is always possible by the choice of $(G, R))$. Define a partial order on $T^{\prime}$ as an extension of $T$ by the following:

(iii') $\operatorname{Min}(c)=\left\{g_{1}, g_{2}, g_{3}\right\}$;

(iv') $\operatorname{Min}(d)=\left\{g_{4}, g_{5}, g_{6}\right\}$;

$\left(\mathrm{v}^{\prime}\right) \operatorname{Min}(e)=\left\{g_{7}, g_{8}, g_{9}\right\}$

$\left(\mathrm{vi}^{\prime}\right) \operatorname{Min}(f)=\left\{g_{10}, g_{11}, g_{12}\right\}$.

Thus, for the graph $(G, R), T$ satisfies $\left(\mathrm{i}^{\prime}\right),\left(\mathrm{ii}^{\prime}\right)$ and $T^{\prime}$ satisfies $\left(\mathrm{i}^{\prime}\right)-\left(\mathrm{vi}^{\prime}\right)$. Clearly, both $T$ and $T^{\prime}$ are triple orders.

For posets $P_{1}$ and $P_{2}$, a map $\varphi: P_{1} \rightarrow P_{2}$ is a constant iff, for $x, y \in P_{1}$, $\varphi(x)=\varphi(y)$.

LEMMA 4.1. Let $\varphi: T \rightarrow T^{\prime}$ be a p-map. Then either $\varphi$ is the inclusion map or a constant. 
ProOF. Consider the case that $\varphi$ is not a constant: it is to be shown that $\varphi$ is the inclusion.

Assume first that there exist distinct $x, y \in G$ such that $\varphi(x)=\varphi(y)$. Then either $\{x, y\} \in R$ or $\{x, y\} \in R^{\prime}$. Suppose $\{x, y\} \in R$. Then since $\varphi$ is a $p$-map and $T^{\prime}$ is a triple order, $\varphi(a)=\varphi(x)=\varphi(y)$. Hence, $\varphi(z)=\varphi(x)=\varphi(a)$ for $\{x, z\} \in R$. Since $(G, R)$ is connected, it follows that $\varphi(u)=\varphi(a)$ for $u \in G$. Further, $\{u, v\} \in R^{\prime}$ for some $u, v \in G$. Whence $\varphi(b)=\varphi(a)$. Consequently, since $T^{\prime}$ is a triple order, it follows that $\varphi$ is a constant. A similar argument holds in the case $\{x, y\} \in R^{\prime}$.

Suppose $\varphi(x)=\varphi(a)$ or $\varphi(b)=\varphi(x)$ for some $x \in G$. Consider $\varphi(a)=\varphi(x)$. For some $y \in G,\{x, y\} \in R$. Thus, $\varphi(a)=\varphi(x)=\varphi(y)$ and, by the above, $\varphi$ is a constant. The case $\varphi(b)=\varphi(x)$ is similar.

Thus $\varphi$ is one-to-one on $G \cup\{a\}$ and on $G \cup\{b\}$.

Suppose next that $\varphi(a) \in G$ or $\varphi(b) \in G$. Consider $\varphi(a) \in G$. Then let $H=\{x \in G: \varphi(x) \in G\}$. Since $\varphi$ is one-to-one on $G,|H|+2 \geq|G|$. Thus, by the choice of $(G, R),|S| \geq 5$ for the induced subgraph $(H, S)$. For $s \in S \subseteq R$, $\varphi(\operatorname{Min}(s)) \subseteq G$. Since $\varphi$ is one-to-one on $H \cup\{a\}$ this contradicts the fact that $\left\{x \in T^{\prime}: x \notin \operatorname{Min}\left(T^{\prime}\right)\right.$ and $\left.\operatorname{Min}(x) \subseteq G\right\}=\{c, d, e, f\}$. Hence $\varphi(a) \notin G$. Similarly, $\varphi(b) \notin G$.

Thus $\varphi(\{a, b\}) \subseteq\{a, b\}$.

Let $\varphi(x) \in\{a, b\}$ for some $x \in G$. By the above, $\varphi(a) \neq \varphi(x)$. Hence, $\{\varphi(a), \varphi(x)\}=\{a, b\}$. For some $y \in G,\{x, y\} \in R$. Since $a, x \in \operatorname{Min}(\{x, y\})$, $a, b \in \operatorname{Min}(\varphi(\{x, y\}))$. This is impossible as no element of $T^{\prime}$ covers both $a$ and $b$. Therefore $\varphi(G)=G$.

Suppose that either $\varphi(a)=a$ or $\varphi(b)=b$. Let $\varphi(a)=a$. Since $\varphi(R) \subseteq R$ and $(G, R)$ is finite, $\varphi$ is a graph automorphism of $G$. By choice, $\varphi$ is the identity on $G$. However, for some $x, y \in G,\{x, y\} \in R^{\prime}$. Thus, $\varphi(b) \neq a$. Hence, $\varphi(b)=b$ and $\varphi$ is the inclusion map. A similar argument applies if $\varphi(b)=b$.

Consider $\varphi(a)=b$ and $\varphi(b)=a$. In this instance, since $\varphi$ is one-to-one on $G \cup\{a, b\}$ and $T^{\prime}$ is a finite triple order, $\varphi(R)=R^{\prime}$ and $\varphi\left(R^{\prime}\right)=R$. That is, $(G, R) \cong\left(G, R^{\prime}\right)$ which is impossible. This covers all outcomes. The proof is complete.

Since $T$ is finite, when endowed with the discrete topology it corresponds to the dual space of a finite pseudocomplemented distributive lattice $L \in B_{3}$. By Lemma 4.1, $\operatorname{End}(L)$ consists of constant endomorphisms (of which there are $|G|+2$ ) and the identity; that is to say, $\operatorname{End}(L)$ has an identity and $|G|+2$ right zeros. It follows from Z. Hedrlín and A. Pultr [25] (see also A. Pultr and V. Trnková [43]) that for any integer $n$ there exist finite graphs $\left(G_{i}: i<n\right)$ satisfying the required conditions (i)-(v) such that, for distinct $i, j<n,\left|G_{i}\right|=\left|G_{j}\right|$ and $G_{i} \not \equiv G_{j}$. The following proposition is immediate.

Proposition 4.2. For any integer $n$ there exist finite pseudocomplemented distributive lattices $\left(L_{i} \in B_{3}: i<n\right)$ and an integer $N$ such that, for distinct $i, j<n, L_{i} \neq L_{j}$ and $\operatorname{End}\left(L_{i}\right) \cong \operatorname{End}\left(L_{j}\right)$ consist of an identity and $N$ right zeros.

Recall that $T^{p}$ denotes the category of compact tôtally order disconnected spaces with the $p$-property together with all continuous $p$-maps and that this category is dually isomorphic to the variety of pseudocomplemented distributive lattices. Let 
$T_{3}^{p}$ denote the full subcategory of $T^{p}$ corresponding to the variety $B_{3}$. Thus, by Proposition 2.1, a compact totally order disconnected space with the $p$-property $(P, \tau)$ is in $T_{3}^{p}$ iff, for $x \in P,|\operatorname{Min}(x)| \leq 3$.

Let $L_{\tau}(2)$ denote the category whose objects are simultaneously $(0,1)$-lattices with two additional unary operations and compact totally disconnected spaces for which the algebraic operations are continuous. (A topological space $(X, \tau)$ is totally disconnected iff, for distinct $x, y \in X$, there exists a clopen set $Y \subseteq X$ such that $x \notin Y$ and $y \in Y$.) Thus an object of $L_{\tau}(2)$ is of the form $(L ; \tau, \vee, \wedge, \alpha, \beta, 0,1)$, where $(L ; \vee, \wedge, \alpha, \beta, 0,1)$ is a $(0,1)$-lattice with two unary operations $\alpha, \beta$ (that is to say, of type $(2,2,1,1,0,0))$ and $(L, \tau)$ is a compact totally disconnected space; furthermore, the algebraic operations are continuous functions. Morphisms of $L_{\tau}(2)$ are all continuous homomorphisms; in other words, all $(0,1)$-lattice homomorphisms preserving $\alpha, \beta$ which are also continuous.

The initial objective is to construct a functor $\Phi$ from $L_{\tau}(2)$ to $T_{3}^{p}$ showing that $L_{\tau}(2)$ is isomorphic to a subcategory of $T_{3}^{p}$ consisting of all nonconstant continuous $p$-maps. Subsequently, a functor $\Psi$ will be given and used to show that the category $A(2)$ of all algebras with two unary operations and their homomorphisms is dually isomorphic to a full subcategory of $L_{\tau}(2)$. A well-known result by Z . Hedrlín and A. Pultr (see $[\mathbf{4 3}]$ ) states that $A(2)$ is universal. A category $C$ is universal if the category of all graphs and their compatible maps is isomorphic to a full subcategory of $C$. Since $T_{3}^{p}$ is dually isomorphic with $B_{3}$, the composite $\Phi \circ \Psi$ together with this duality will establish the almost universality of $B_{3}$.

Construction 4.3. Define a functor $\Phi: L_{\tau}(2) \rightarrow T_{3}^{p}$ as follows:

For $(L ; \tau, \vee, \wedge, \alpha, \beta, 0,1) \in L_{\tau}(2)$, let $P$ be the disjoint union

$\{a, b\} \cup\left(L \times\left(G \cup R \cup R^{\prime}\right)\right) \cup((L \times L) \times\{0,1\}) \cup L \cup\{g\}$, where $G, R, R^{\prime}$ and $\{a, b\}$ are as before.

Define a partial order on $P$ such that the following hold:

(i) for $x, y \in L$ and $s, t \in G \cup R \cup R^{\prime},(x, s) \geq(y, t)$ in $P$ iff $x=y$ and $s \geq t$ in $T$;

(ii) for $x \in L, s \in G \cup R \cup R^{\prime}$ and $t \in\{a, b\},(x, s) \geq t$ in $P$ iff $s \geq t$ in $T$;

(iii) for $(x, y) \in L \times L, \operatorname{Min}((x, y, 0))=\left\{\left(x, g_{1}\right),\left(y, g_{2}\right),\left(x \vee y, g_{3}\right)\right\}$, and $\operatorname{Min}((x, y, 1))=\left\{\left(x, g_{4}\right),\left(y, g_{5}\right),\left(x \wedge y, g_{6}\right)\right\}$

(iv) for $x \in L, \operatorname{Min}(x)=\left\{\left(x, g_{7}\right),\left(\alpha(x), g_{8}\right),\left(\beta(x), g_{9}\right)\right\}$;

(v) $\operatorname{Min}(g)=\left\{\left(0, g_{10}\right),\left(0, g_{11}\right),\left(1, g_{12}\right)\right\}$.

Intuitively, $P$ contains $L$ copies of $T$ where the elements $a$ and $b$ are identified; all remaining elements reflect the algebraic operations of $(L ; \vee, \wedge, \alpha, \beta, 0,1)$.

Define a topology on $P$ in the following manner. $P$ is the union of finitely many sets: two singletons $\{a\},\{b\} ;\left|G \cup R \cup R^{\prime}\right|$ copies of $L$; two copies of $L \times L$; a single copy of $L$; a singleton $\{g\}$. Since $(L, \tau)$ is a compact totally disconnected space, so is $L \times L$ in the product topology. Let $\sigma$ be the union topology on $P$; more precisely, $(P, \sigma)$ is the union of three isolated points, $\left|G \cup R \cup R^{\prime}\right|+1$ copies of $L$ with topology $\tau$, and two copies of $L \times L$ with the product topology. It immediately follows that $(P, \sigma)$ is a compact totally disconnected space.

Let $\Phi(L ; \tau, \vee, \wedge, \alpha, \beta, 0,1)=(P, \sigma)$.

For $L_{1}, L_{2} \in L_{\tau}(2)$, let $\psi: L_{1} \rightarrow L_{2}$ be a continuous homomorphism. Define $\Phi(\psi): \Phi\left(L_{1}\right) \rightarrow \Phi\left(L_{2}\right)$ as follows:

(i) $\Phi(\psi)(a)=a$;

(ii) $\Phi(\psi)(b)=b$; 
(iii) for $x \in L_{1}$ and $s \in G \cup R \cup R^{\prime}, \Phi(\psi)(x, s)=(\psi(x), s)$;

(iv) for $x, y \in L_{1}$ and $i=0,1, \Phi(\psi)(x, y, i)=(\psi(x), \psi(y), i)$;

(v) for $x \in L_{1}, \Phi(\psi)(x)=\psi(x)$;

(vi) $\Phi(\psi)(g)=g$.

It must be shown that $\Phi$ is the well-defined functor that it is claimed to be.

Initially it is to be shown that $\Phi(L) \in T_{3}^{p}$ for all $L \in L_{\tau}(2)$.

The first lemma is routine.

LEMMA 4.4. For $L \in L_{\tau}(2), \Phi(L)$ is a triple order. $L_{\tau}(2)$.

LEMMA 4.5. $\Phi(L)$ is a compact totally order disconnected space for any $L \in$

Proof. Clearly, as defined, for $L \in L_{\tau}(2), \Phi(L)$ is a compact totally disconnected space.

For $p, q \in P$, let $p \not \leq q$. Then it must be shown that there is a clopen decreasing set $Q \subseteq P$ such that $q \in Q$ and $p \notin Q$.

Suppose $p=(x, s)$ and $q=(y, t)$ for $x, y \in L$ and $s, t \in G \cup R \cup R^{\prime}$. If $x \neq y$ then there exists a clopen set $U \subseteq L$ such that $x \notin U$ and $y \in U$. Thus, for $Q=U \times\{r: r \leq t\} \cup\{a, b\}, q \in Q, p \notin Q$, and $Q$ is clopen decreasing. If $x=y$ then $s \leq t$; set $Q=L \times\{r: r \leq t\} \cup\{a, b\}$.

If either $p$ or $q$ is $a, b$ or $g$, then the construction of $Q$ is clear. Similarly, if $p \in L$ or $(L \times L) \times\{0,1\}$, then finding a suitable $Q$ is again straightforward.

Thus it remains to consider the cases for which $q \in L$ or $q \in(L \times L) \times\{0,1\}$. Of these the cases that bear closer inspection are when $p=\left(x, g_{i}\right)$ for $i=1,2,3$ and $q \in(L \times L) \times\{0\}$, or $p=\left(x, g_{i}\right)$ for $i=4,5,6$ and $q \in(L \times L) \times\{1\}$, or $p=\left(x, g_{i}\right)$ for $i=7,8,9$ and $q \in L$. Suppose first that $q \in(L \times L) \times\{0\}$ and $p=\left(x, g_{i}\right)$ for $i=1,2$, or 3 . Consider the unique element $\left(y, g_{i}\right)$ such that $q>\left(y, g_{i}\right)$. Since $x \neq y$, choose a clopen set $U \subseteq L$ such that $x \notin U$ and $y \in U$. Let $V=\left\{r \in(L \times L) \times\{0\}: r>\left(z, g_{i}\right)\right.$ for some $\left.z \in U\right\}$. Then $V$ is clopen since, in the case $i=1,2$, projections are continuous and, in the case $i=3$, the join function is continuous by hypothesis. Let $Q=V \cup\left(U \times\left\{g_{i}\right\}\right) \cup\left(L \times\left\{g_{j}: j \neq i\right.\right.$ and $j=1,2,3\})$. For $q \in(L \times L) \times\{1\}$, a similar argument holds. Finally, let $p=\left(x, g_{i}\right)$ for $i=7,8,9$, and let $q \in L$. Then there is exactly one $\left(y, g_{i}\right)$ such that $q>\left(y, g_{i}\right)$. Similarly, since $x \neq y$, there exists a clopen set $U \subseteq L$ such that $y \in U$ and $x \notin U$. Because $\alpha, \beta$ are continuous, $V=\left\{r \in L: r>\left(z, g_{i}\right)\right.$ for some $\left.z \in U\right\}$ is clopen. It is then sufficient to consider $Q=V \cup\left(U \times\left\{g_{i}\right\}\right) \cup\left(L \times\left\{g_{j}: j \neq i\right.\right.$ and $j=7,8,9\})$. The proof is complete.

LEMMA 4.6. For $L \in L_{\tau}(2), \Phi(L)$ has the p-property.

ProOF. It must be shown that $[Q]$ is clopen for any clopen decreasing set $Q \subseteq P$. However, a subset of $P$ is clopen iff its intersection with each copy of $L$ or $L \times L$ is clopen. The claim follows by observing, as in the previous lemma, that the projections, join, meet, $\alpha$ and $\beta$ are all continuous functions.

Thus, for $L \in L_{\tau}(2), \Phi(L) \in T_{3}^{p}$. The next lemma is routine.

LEMMA 4.7. For $L_{1}, L_{2} \in L_{\tau}(2)$ and a continuous homomorphism $\psi: L_{1} \rightarrow$ $L_{2}, \Phi(\psi)$ is a continuous p-map. 
Consequently, $\Phi$ is a well-defined faithful functor. It is now to be shown that it is almost full; that is to say, if, for $L_{1}, L_{2} \in L_{\tau}(2), \varphi: \Phi\left(L_{1}\right) \rightarrow \Phi\left(L_{2}\right)$ is a continuous $p$-map which is not a constant, then there exists a continuous homomorphism $\psi: L_{1} \rightarrow L_{2}$ such that $\varphi=\Phi(\psi)$.

LEMMA 4.8. For $L_{1}, L_{2} \in L_{\tau}(2)$, let $\varphi: \Phi\left(L_{1}\right) \rightarrow \Phi\left(L_{2}\right)$ be a continuous $p$ map which is not a constant. Then $\varphi(a)=a, \varphi(b)=b$, and there exists a mapping $\psi: L_{1} \rightarrow L_{2}$ such that $\varphi(x, s)=(\psi(x), s)$ for all $x \in L_{1}$ and $s \in G \cup R \cup R^{\prime}$.

Proof. For $x \in L_{1}$, let $\xi_{x}: T \rightarrow \Phi\left(L_{1}\right)$ be the mapping defined as follows:

(i) $\xi_{x}(a)=a$;

(ii) $\xi_{x}(b)=b$;

(iii) for $s \in G \cup R \cup R^{\prime}, \xi_{x}(s)=(x, s)$.

Let $\xi: \Phi\left(L_{2}\right) \rightarrow T^{\prime}$ be the mapping defined by

(i) $\xi(a)=a$;

(ii) $\xi(b)=b$;

(iii) for $x \in L_{2}$ and $s \in G \cup R \cup R^{\prime}, \xi(x, s)=s$;

(iv) for $x, y \in L_{2}, \xi(x, y, 0)=c, \xi(x, y, 1)=d$;

(v) for $x \in L_{2}, \xi(x)=e$;

(vi) $\xi(g)=f$.

Clearly, for $x \in L_{1}, \xi_{x}: T \rightarrow \Phi\left(L_{1}\right), \varphi: \Phi\left(L_{1}\right) \rightarrow \Phi\left(L_{2}\right)$, and $\xi: \Phi\left(L_{2}\right) \rightarrow T^{\prime}$ are all $p$-maps. Let $\rho_{x}: T \rightarrow T^{\prime}$ denote the $p$-map $\xi \circ \varphi \circ \xi_{x}$. By Lemma 4.1, $\rho_{x}$ is a constant or inclusion. Hence, it is either the case that $\rho_{x}$ is a constant for some $x \in L_{1}$ or that $\rho_{x}$ is the inclusion for every $x \in L_{1}$.

Suppose, for some $x \in L_{1}, \rho_{x}$ is a constant. Since $\rho_{x}$ is a $p$-map, either $\varphi \circ \xi_{x}(T)=$ $\{a\}, \varphi \circ \xi_{x}(T)=\{b\}$, or, for some $s \in G, \varphi \circ \xi_{x}(T) \subseteq L_{2} \times\{s\}$. However, $L_{2} \times\{s\}$ is an antichain in $\Phi\left(L_{2}\right)$. Thus, in either case, $\varphi \circ \xi_{x}$ is a constant. Since, for $y \in L_{1}$, $\rho_{y}(a)=\rho_{x}(a)$ and $\rho_{y}(b)=\rho_{x}(b), \rho_{y}$ is a constant for all $y \in L_{1}$ and, consequently, $\varphi \circ \xi_{y}=\varphi \circ \xi_{x}$ for all $y \in L_{1}$. From the fact that $\operatorname{Min}\left(\Phi\left(L_{1}\right)\right) \subseteq \bigcup\left(\xi_{y}(T): y \in L_{1}\right)$, it follows that $\varphi$ is a constant on $\operatorname{Min}\left(\Phi\left(L_{1}\right)\right)$. Since $\Phi\left(L_{2}\right)$ is a triple order, $\varphi$ must be a constant on $\Phi\left(L_{1}\right)$. By hypothesis, this is impossible.

Consider the alternative: $\rho_{x}$ is the inclusion for every $x \in L_{1}$. In particular, $\varphi(a)=a, \varphi(b)=b$, and, for $x \in L_{1}$ and $s \in G$, there exists $y \in L_{2}$ such that $\varphi(x, s)=(y, s)$. Choose $t \in G$. Then either $\{s, t\} \in R$ or $\{s, t\} \in R^{\prime}$. Whence, $\operatorname{Min}((x,\{s, t\}))=\{a,(x, s),(x, t)\}$ or $\{b,(x, s),(x, t)\}$. Since $\varphi(a)=a, \varphi(b)=b$, and $\varphi(x, s)=(y, s)$, it follows that $\varphi(x, t)=(y, t)$ and $\varphi(x,\{s, t\})=(y,\{s, t\})$. Let $\psi(x)=y$. The proof is complete.

LEMMA 4.9. For $L_{1}, L_{2} \in L_{\tau}(2)$, let $\varphi: \Phi\left(L_{1}\right) \rightarrow \Phi\left(L_{2}\right)$ be a continuous $p$-map which is not a constant. Then there exists a continuous homomorphism $\psi: L_{1} \rightarrow L_{2}$ such that $\varphi=\Phi(\psi)$.

PrOOF. By Lemma 4.8, there is a mapping $\psi: L_{1} \rightarrow L_{2}$ such that $\varphi(a)=a$, $\varphi(b)=b$, and $\varphi(x, s)=(\psi(x), s)$ for every $x \in L_{1}$ and $s \in G \cup R \cup R^{\prime}$. Clearly, $\psi$ is continuous. It must be shown that $\psi$ is a homomorphism.

For $x, y \in L_{1}, \operatorname{Min}((x, y, 0))=\left\{\left(x, g_{1}\right),\left(y, g_{2}\right),\left(x \vee y, g_{3}\right)\right\}$. Hence, $\operatorname{Min}(\varphi(x, y, 0))$ $=\left\{\left(\psi(x), g_{1}\right),\left(\psi(y), g_{2}\right),\left(\psi(x \vee y), g_{3}\right)\right\}$. In order that a point with these three minimals exist it is necessary that $\psi(x \vee y)=\psi(x) \vee \psi(y)$; whence, $\varphi(x, y, 0)=$ $(\psi(x), \psi(y), 0)$. Thus $\psi$ preserves joins and $\varphi$ has the required form on $L_{1} \times L_{1} \times\{0\}$. 
A similar argument shows that $\psi$ preserves meets and that $\varphi$ has the required form on $L_{1} \times L_{1} \times\{1\}$.

For $x \in L_{1}, \operatorname{Min}(x)=\left\{\left(x, g_{7}\right),\left(\alpha(x), g_{8}\right),\left(\beta(x), g_{9}\right)\right\}$. Hence, $\operatorname{Min}(\varphi(x))=$ $\left\{\left(\psi(x), g_{7}\right),\left(\psi(\alpha(x)), g_{8}\right),\left(\psi(\beta(x)), g_{9}\right)\right\}$. Such a point exists iff $\psi(\alpha(x))=\alpha(\psi(x))$ and $\psi(\beta(x))=\beta(\psi(x))$; in which case, $\varphi(x)=\psi(x)$. Thus $\varphi$ has the required form for $L_{1}$ and $\psi$ preserves the unary operations.

Finally, since $\operatorname{Min}(\varphi(g))=\left\{\left(\psi(0), g_{10}\right),\left(\psi(0), g_{11}\right),\left(\psi(1), g_{12}\right)\right\}$, it follows that $\varphi(g)=g$ and, hence, $\psi$ preserves 0 and 1 .

Consequently, $\psi: L_{1} \rightarrow L_{2}$ is a continuous homomorphism and $\varphi=\Phi(\psi)$. The proof is complete.

The above results combine to give the following

THEOREM 4.10. $\Phi: L_{\tau}(2) \rightarrow T_{3}^{p}$ is an almost full embedding.

The objective now is to construct a contravariant functor $\Psi$ from $A(2)$, the variety of algebras with two unary operations, to a full subcategory of $L_{\tau}(2)$. By means of $\Psi$ it will be shown that $A(2)$ is dually isomorphic to a full subcategory of $L_{\tau}(2)$.

For a set $X$, let $B(X)$ denote the set of all subsets of $X$. There is a natural identification between $B(X)$ and $2^{X}$; namely, any subset of $X$ corresponds to its characteristic function. For every $f: X \rightarrow Y$ let $B(f): B(Y) \rightarrow B(X)$ be defined by $B(f)(Z)=f^{-1}(Z)$. Under the above identification the $x$ th projection $\pi_{x}: 2^{X} \rightarrow 2$ is just $B\left(\xi_{x}\right)$ for the mapping $\xi_{x}: 1 \rightarrow X$ with the value $x \in X$. The topology $\tau_{X}$ of the product $B(X)=2^{X}$ of $X$ copies of the discrete space 2 is thus generated by all maps $B\left(\xi_{x}\right)=\pi_{x}$ with $x \in X$. The following proposition is well known (see, for example, J. L. Kelley [29]).

LEMMA 4.11. $\left(B(X), \tau_{X}\right)$ is a compact totally disconnected space for any set $X$.

For any set $X, B(X)$ also is a $(0,1)$-lattice where union and intersection represent join and meet. Thus $\left(B(X) ; \tau_{X}, \vee, \wedge, 0,1\right)$ is simultaneously a bounded lattice $(B(X) ; \vee, \wedge, 0,1)$ and a compact totally disconnected space $\left(B(X), \tau_{X}\right)$. For every $f: X \rightarrow Y$, the lattice $(0,1)$-homomorphism $B(f)$ is continuous: $\tau_{X}$ has a subbase consisting of sets of the form $B\left(\xi_{x}\right)^{-1}\{i\}$ for $i=0,1$ and $x \in X$, and $B\left(\xi_{x}\right) \circ B(f)=B\left(\xi_{f(x)}\right)$ for every $x \in X$.

The two claims below follow by $[\mathbf{1 2}$ and 14]; their proofs are included here for the sake of completeness.

LEMMA 4.12. The binary operations $\vee, \wedge$ are $\tau_{X}$-continuous.

Proof. Let $J(A, B)=A \cup B$ for $A, B \subseteq X$; we aim to show that $J$ is continuous with respect to the product topology on $B(X)^{2}$. Let $p_{i}: B(X)^{2} \rightarrow B(X)$ be the two projections for $i=0,1$. Clearly $B\left(\xi_{x}\right)(J(A, B))=0$ is equivalent to $x \in X \backslash(A \cup B)$ and the latter statement holds if and only if $B\left(\xi_{x}\right) p_{0}(A, B)=B\left(\xi_{x}\right) p_{1}(A, B)=0$. Since $p_{0}, p_{1}$, and all $B\left(\xi_{x}\right)$ are continuous, $J^{-1}\left(B\left(\xi_{x}\right)^{-1}\{0\}\right)$ is clopen in $B(X)^{2}$ for every $x \in X$. Since the maps $B\left(\xi_{x}\right)$ generate $\tau_{X}$, this proves that $J$ is continuous. The continuity of meet easily follows.

LEMMA 4.13. $\psi: B(Y) \rightarrow B(X)$ is a continuous lattice $(0,1)$-homomorphism if and only if $\psi=B(\xi)$ for some mapping $\xi: X \rightarrow Y$. 
The proof of Lemma 4.13 will be facilitated by the lemma below. Before its formulation, recall that $\left(B(1) ; \tau_{1}, \vee, \wedge, 0,1\right)$ is simply the two-element chain $2=$ $\{0,1\}$ with $0<1$, endowed with the discrete topology.

LEMMA 4.14. A mapping $\gamma$ of $\left(B(X) ; \tau_{X}, \vee, \wedge, 0,1\right)$ into $B(1)$ is a continuous lattice $(0,1)$-homomorphism if and only if $\gamma=B\left(\xi_{x}\right)$ for some $x \in X$.

PROOF. If $\gamma$ is a continuous lattice $(0,1)$-homomorphism, then $\gamma^{-1}\{1\}$ is a $\tau_{X^{-}}$ clopen set containing $X$. Since $\tau_{X}$ is generated by the system $\left(B\left(\xi_{x}\right): x \in X\right)$, the set $\gamma^{-1}\{1\}$ is a union of (finitely many) basic sets and there exists a finite $F \subseteq X$ such that $X$ is an element of a basic set $A=\bigcap\left(B\left(\xi_{x}\right)^{-1}\left\{i_{x}\right\}: x \in F\right)$ contained in $\gamma^{-1}\{1\}$; here $i_{x} \in\{0,1\}$ for every $x \in F$. In fact, $i_{x}=1$ must hold for all $x \in F$ because $X \in A$. It follows that $A$ is the family of all $Z \subseteq X$ such that $F \subseteq Z$. In particular, $F \in A \subseteq \gamma^{-1}\{1\}$; since $F$ is the join of all singletons $\{x\}$ with $x \in F$ and $\gamma$ is a lattice homomorphism, there is an $x \in F$ with $\gamma(\{x\})=1$. If $x \in Z \subseteq X$, then $\gamma(Z)=\gamma(\{x\}) \vee \gamma(Z)=1$, while $x \notin Z$ yields $\gamma(Z)=\gamma(Z) \wedge 1=\gamma(Z \wedge\{x\})=\gamma(\varnothing)=0$; in other words, $\gamma=B\left(\xi_{x}\right)$. This completes the proof since the converse is trivial.

ProOF OF LEMMA 4.13. Suppose $\psi: B(Y) \rightarrow B(X)$ is a continuous lattice $(0,1)$-homomorphism. For every $x \in X$ the composite $B\left(\xi_{x}\right) \circ \psi=B\left(\xi_{y}\right)$ for a unique $y \in Y$, by Lemma 4.14. Set $\xi(x)=y$ and note that $x \in \psi(Z)$ is equivalent to $x \in B\left(\xi_{y}\right)(Z)$; in other words, $\psi(Z)=B(\xi)(Z)$ for all $Z \subseteq Y$. Hence $\psi=B(\xi)$ and the proof is complete.

Thus, for a set $X,\left(B(X) ; \tau_{X}, \vee, \wedge, 0,1\right)$ is composed of a compact totally disconnected space $\left(B(X), \tau_{X}\right)$ (Lemma 4.11) and a $(0,1)$-lattice $(B(X) ; \vee, \wedge, 0,1)$ for which the operations $\vee, \wedge$ are continuous (Lemma 4.12). By Lemma 4.13, continuous $(0,1)$-lattice endomorphisms of $B(X)$ are exactly all maps of the form $B(\xi)$ for some $\xi: X \rightarrow X$. If $(X ; \alpha, \beta)$ is an algebra with two unary operations $\alpha, \beta$, then $\Psi(X ; \alpha, \beta)=\left(B(X) ; \tau_{X}, \vee, \wedge, B(\alpha), B(\beta), 0,1\right)$ is an object of $L_{\tau}(2)$. Let $B_{\tau}(2)$ be the category of all such objêcts together with all continuous homomorphisms. Thus $B_{\tau}(2)$ is a full subcategory of $L_{\tau}(2)$.

Recall that $\xi:(X ; \alpha, \beta) \rightarrow(Y ; \alpha, \beta)$ is a homomorphism in $A(2)$ if and only if $\xi \circ \alpha=\alpha \circ \xi$ and $\xi \circ \beta=\beta \circ \xi$. It is easily seen that these equalities hold if and only if $B(\alpha) \circ B(\xi)=B(\xi) \circ B(\alpha)$ and $B(\beta) \circ B(\xi)=B(\xi) \circ B(\beta)$. Setting $\Psi(\xi)=B(\xi)$ for any morphism $\xi$ in $A(2)$ gives rise to a contravariant full embedding $\Psi: A(2) \rightarrow L_{\tau}(2)$ whose range is the full subcategory $B_{\tau}(2)$ of $L_{\tau}(2)$. This concludes the proof of the claim below.

THEOREM 4.15. $A(2)$ is dually isomorphic to $B_{\tau}(2)$.

Since $A(2)$ is universal and $T_{3}^{p}$ is dually isomorphic to $B_{3}$, Theorems 4.10 and 4.15 combine to prove the following

THEOREM 4.16. The variety $B_{3}$ is almost universal.

COROLlaRY 4.17. The varieties $B_{4}, \ldots, B_{\omega}$ are almost universal.

Any almost universal variety is isomorphism universal. Adding a new zero to any distributive $(0,1)$-lattice produces a pseudocomplemented lattice in the variety $B_{1}$. Since any lattice isomorphism preserves all existing pseudocomplements, the following is an immediate consequence of Theorem 4.13. 
COROLLARY 4.18. The variety $B_{1}$ of Stone algebras is isomorphism universal.

This also strengthens Birkhoff's representation theorem [9] by showing that there is a proper class of nonisomorphic distributive lattices whose automorphism groups are isomorphic to a given group. In fact, the presented construction implies the existence of the maximal possible number of nonisomorphic such distributive lattices of a given infinite cardinality not smaller than that of the represented group.

Let $\kappa$ be an infinite cardinal. It follows from [26] that, for any monoid $M$ of cardinality $\leq \kappa$, there are algebras $\left(X_{i} \in A(2): i<2^{\kappa}\right)$ such that, for $i<2^{\kappa}$, $\left|X_{i}\right|=\kappa, \operatorname{End}\left(X_{i}\right) \cong M$, and, for distinct $j<2^{\kappa}$, there are no homomorphisms $\xi: X_{i} \rightarrow X_{j}$. Under the construction, for $i<2^{\kappa}, \Psi\left(X_{i}\right)$ has $2^{\kappa}$ minimal elements. Thus, in particular,

THEOREM 4.19. Let $M$ be a monoid such that $|M| \leq \kappa$, where $\kappa$ is an infinite cardinal. Then there exists a system $\left(L_{i} \in B_{3}: i<2^{\kappa}\right)$ such that, for $i<2^{\kappa}$, $\left|L_{i}\right|=\kappa$, the nonconstant endomorphisms of $L_{i}$ form a monoid isomorphic to $M$, and $\operatorname{Hom}\left(L_{i}, L_{j}\right)$ consists of constants whenever $i \neq j$. In particular, if $M$ is trivial, then $\operatorname{End}\left(L_{i}\right) \cong \operatorname{End}\left(L_{j}\right)$ consists of $2^{\kappa}$ right zeros and a unit.

Moreover, if $M$ is finite, then, for $N<\omega$, there is a family $\left(L_{i} \in B_{3}: i<N\right)$ such that, for distinct $i, j<N,\left|L_{i}\right|=\left|L_{j}\right|<\aleph_{0}$, the nonconstant endomorphisms of $L_{1}$ are isomorphic to $M$, and if $f: L_{i} \rightarrow L_{j}$ is a homomorphism then it is a constant.

5. Heyting algebras. The objective now is to extend the results of the last section to the variety of Heyting algebras.

Since every Heyting algebra is a distributive $(0,1)$-lattice, it is representable by a compact totally order disconnected topological space. Any homomorphism $h: H \rightarrow H^{\prime}$ of Heyting algebras is a $(0,1)$-homomorphism and hence it corresponds to a unique continuous order preserving mapping of the space representing $H^{\prime}$ to that representing $H$. The next two lemmas are part of the folklore (see, for instance, H. A. Priestley [42]).

LEMMA 5.1. Let $P$ be a compact totally order disconnected topological space. Then $P$ is a dual space of a Heyting algebra if and only if $[Q)$ is clopen for every convex clopen $Q \subseteq P$ (that is, if $P$ has the h-property).

LEMMA 5.2. Let $\varphi: P \rightarrow P^{\prime}$ be a continuous map and let $P, P^{\prime}$ be compact totally order disconnected spaces with the h-property. Then $\varphi$ is the dual of a Heyting homomorphism if and only if it preserves order and $\varphi((x])=(\varphi(x)]$ for every $x \in P$ (that is, if $\varphi$ is an h-map).

The variety of all Heyting algebras is thus dually isomorphic to the category $T^{h}$ of all continuous $h$-maps between compact totally order disconnected topological spaces with the $h$-property.

Recall that the functor $\Phi$ constructed in $\S 4$ is such that $\Phi(L)$ is a triple order and $\operatorname{Min}(\Phi(L))$ is a clopen set for every $L \in L_{\tau}(2)$. Hence $Q \cap \operatorname{Min}(\Phi(L))$ is a clopen set for any clopen $Q \subseteq \Phi(L)$. Furthermore, $[Q \cap \operatorname{Min}(\Phi(L)))$ is clopen by the $p$-property of $\Phi(L)$; since $\Phi(L)$ is a triple order, $[Q)=Q \cup[Q \cap \operatorname{Min}(\Phi(L)))$. Whence $[Q)$ is a clopen set. That is, $\Phi(L)$ has the $h$-property for every $L \in L_{\tau}(2)$. Now if $\varphi: \Phi(L) \rightarrow \Phi\left(L^{\prime}\right)$ is an order preserving $p$-map then it also is an $h$-map 
because $(\varphi(x)]=\operatorname{Min}(\varphi(x)) \cup\{f(x)\}$ in the triple order of $\Phi\left(L^{\prime}\right)$; it is clear that, conversely, every $h$-map is a $p$-map.

These remarks together with Theorems 4.10 and 4.15 show that Heyting algebras form an almost universal variety as claimed by Theorem 1.2.

There is a consequence analogous to Theorem 4.19.

THEOREM 5.3. Let $\kappa$ be an infinite cardinal and let $M$ be a monoid with no more than $\kappa$ elements. Then there is a family $\left(H_{i}: i<2^{\kappa}\right)$ of Heyting algebras such that $\left|H_{i}\right|=\kappa$ for all $i<2^{\kappa}$, the nonconstant endomorphisms of each $H_{i}$ form a monooid isomorphic to $M$, and $\operatorname{Hom}\left(H_{i}, H_{j}\right)$ consists of constants for distinct $i, j<2^{\kappa}$. Furthermore, each $H_{i}$ has exactly $2^{\kappa}$ constant endomorphisms.

\section{REFERENCES}

1. M. E. Adams, Implicational classes of pseudocomplemented distributive lattices, J. London Math. Soc. (2) 13 (1976), 381-384.

2. M. E. Adams, V. Koubek and J. Sichler, Homomorphisms and endomorphisms of distributive lattices, Houston J. Math. (to appear).

3. R. Balbes and Ph. Dwinger, Distributive lattices, University of Missouri Press, Columbia, Missouri, 1974.

4. R. Balbes and G. Grätzer, Injective and projective Stone algebras, Duke Math. J. 38 (1971), 339-347.

5. R. Balbes and A. Horn, Injective and projective Heyting algebras, Trans. Amer. Math. Soc. 148 (1970), 549-560.

6. H. J. Bandelt, Endomorphism semigroups of median algebras, Algebra Universalis 12 (1981), 262-264.

7. __ Isomorphisms between semigroups of isotone maps, J. Austral. Math. Soc. Ser. A 30 (1981), 453-460.

8. R. Beazer, Pseudocomplemented algebras with Boolean congruence lattices, J. Austral. Math. Soc. Ser. A 26 (1978), 163-168.

9. G. Birkhoff, On groups of automorphisms, Rev. Un. Mat. Argentina 11 (1946), 155-157.

10. C. C. Chen and G. Grätzer, Stone lattices. I: Construction theorems, Canad. J. Math. 21 (1969), 884-894.

11. __ Stone lattices. II: Structure theorems, Canad. J. Math. 21 (1969), 895-903.

12. B. A. Davey and D. Duffus, Exponentiation and duality, Ordered Sets, NATO Advanced Study Inst. Series 83, Reidel, Dordrecht, 1982, pp. 43-95.

13. B. A. Davey and M. S. Goldberg, The free p-algebra generated by a distributive lattice, Algebra Universalis 11 (1980), 90-100.

14. G. Gierz, K. H. Hofmann, K. Keimel, J. D. Lawson, M. Mislove and D. S. Scott, A compendium of continuous lattices, Springer-Verlag, Berlin, Heidelberg and New York, 1980.

15. V. Glivenko, Sur quelques points de la logique de M. Browwer, Bull. Acad. Sci. Belgique 15 (1929), 183-188.

16. G. Grätzer, Lattice theory: First concepts and distributive lattices, Freeman, San Francisco, Calif., 1971.

17. _ General lattice theory, Birkhäuser Verlag, Basel and Stuttgart, 1978.

18. G. Grätzer and H. Lakser, The structure of pseudocomplemented distributive lattices. II: Congruence extension and amalgamation, Trans. Amer. Math. Soc. 156 (1971), 343-358.

19. _ , The structure of pseudocomplemented distributive lattices. III: Injective and absolute subretracts, Trans. Amer. Math. Soc. 169 (1972), 475-487.

20. G. Grätzer, H. Lakser and R. W. Quackenbush, On the lattice of quasivarieties of distributive lattices with pseudocomplementation, Acta Sci. Math. (Szeged) 42 (1980), 257-263.

21. G. Grätzer and E. T. Schmidt, On a problem of M. H. Stone, Acta Math. Acad. Sci. Hungar. 8 (1957), 455-460.

22. J. Hashimoto, Ideal theory for lattices, Math. Japon. 2 (1952), 149-186. 
23. Z. Hedrlín and R. H. McDowell, Partly ordered sets with given monoid of order preserving mappings, manuscript.

24. Z. Hedrlín and A. Pultr, Relations (graphs) with given infinite semigroups, Monatsh. Math. 68 (1964), 421-425.

25. __ On rigid undirected graphs, Canad. J. Math. 18 (1966), 1237-1242.

26. Z. Hedrlín and J. Sichler, Any boundable binding category contains a proper class of mutually disjoint copies of itself, Algebra Universalis 1 (1971), 97-103.

27. T. Katrinák, Die Kennzeichnung der distributiven pseudokomplementären Halbverbände, J. Reine Angew. Math. 241 (1970), 160-172.

28. __ Über eine Konstruktion der distributiven pseudokomplementären Verbände, Math. Nachr. 53 (1971), 85-99.

29. J. L. Kelley, General topology, Van Nostrand Reinhold, New York, 1955.

30. P. Köhler, The triple method and free distributive pseudocomplemented lattices, Algebra Universalis 8 (1978), 139-150.

31. __ Endomorphism semigroups of Brouwerian semilattices, Semigroup Forum 15 (1978), 229 234.

32. H. Lakser, The structure of pseudocomplemented distributive lattices. I, Trans. Amer. Math. Soc. 156 (1971), 335-342.

33. K. B. Lee, Equational classes of distributive pseudocomplemented lattices, Canad. J. Math. 22 (1970), 881-891.

34. K. D. Magill, The semigroup of endomorphisms of a Boolean ring, Semigroup Forum 4 (1972), 411-416.

35. C. J. Maxson, On semigroups of Boolean ring endomorphisms, Semigroup Forum 4 (1972), 78-82.

36. R. McKenzie and J. D. Monk, On automorphism groups of Boolean algebras, Erdös Symposium, Colloq. Math. Soc. János Bolyai 10, North-Holland, Amsterdam, 1973, pp. 951-988.

37. R. McKenzie and C. Tsinakis, On recovering a bounded distributive lattice from its endomorphism monoid, Houston J. Math. 7 (1981), 525-529.

38. D. Pigozzi and J. Sichler, Homomorphisms of partial and of complete Steiner triple systems and quasigroups (to appear).

39. H. A. Priestley, Representation of distributive lattices by means of ordered Stone spaces, Bull. London Math. Soc. 2 (1970), 186-190.

40. _ Ordered topological spaces and the representation of distributive lattices, Proc. London Math. Soc. (3) 24 (1972), 507-530.

41. _ The construction of spaces dual to pseudocomplemented distributive lattices, Quart. J. Math. Oxford (2) 26 (1975), 215-228.

42. _ Ordered sets and duality for distributive lattices, Proc. Conf. on Ordered Sets and their Applications (Lyon, 1982) (to appear).

43. A. Pultr and V. Trnková, Combinatorial, algebraic and topological representations of groups, semigroups and categories, North-Holland, Amsterdam, 1980.

44. H. Rasiowa and R. Sikorski, The mathematics of metamathematics, Monogr. Mat., PWN, Warsaw, 1963.

45. P. Ribenboim, Characterization of the sup-complement in a distributive lattice with last element, Summa Brasil. Math. 2 (1949), 43-49.

46. B. M. Schein, Ordered sets, semilattices, distributive lattices and Boolean algebras with homomorphic endomorphism semigroups, Fund. Math. 68 (1970), 31-50.

47. C. Tsinakis, Brouwerian semilattices determined by their endomorphism semigroups, Houston J. Math. 5 (1979), 427-436.

48. A. Urquhart, Projective distributive p-algebras, Bull. Austral. Math. Soc. 24 (1981), 269-275.

49. J. C. Varlet, Contribution à l'etude des treillis pseudocomplémentés et des treillis de Stone, Mém. Soc. Roy. Sci. Liége Coll. 8 (1963).

Department of Mathematics, State University of New York, New Paltz, NEW YORK 12561

MFF KU, Malostranské nám. 25, Praha 1, Czechoslovakia

DePARTMENT of MATHEMAtics, University of Manitoba, WinNipeg, Manitoba, CANADA R3T 2N2 